Nevşehir Bilim ve Teknoloji Dergisi Cilt 4(1) 1-33 2015

DOI: 10.17100/nevbiltek.210937

URL: http://dx.doi.org/10.17100/nevbiltek.210937

\title{
A Study on the Fauna of Heteroptera of Ilgaz Mountains (Kastamonu, Çankırı) With a New Record for Turkey
}

\author{
İbrahim Küçükbasmacı ${ }^{1, *}$, Suat Kıyak ${ }^{2}$ \\ ${ }^{1}$ Kastamonu University, Sciences and Arts Faculty, Department of Biology, Kastamonu/Turkey \\ ${ }^{2}$ Gazi University, Sciences Faculty, Department of Biology, 06500-Teknikokullar, Ankara/Turkey
} Abstract

This research was carried out in the Ilgaz Mountains between May 1999 and September 2000, ninety three Heteroptera species and subspecies belonging to 73 genera of 16 families (Notonectidae, Gerridae, Veliidae, Miridae, Anthocoridae, Reduviidae, Nabidae, Berytidae, Lygaeidae, Pyrrhocoridae, Stenocephalidae, Coreidae, Alydidae, Rhopalidae, Scutelleridae and Pentatomidae) were determined. 75 of 93 taxa are new for the Heteroptera fauna of Ilgaz Mountains. Addition, Velia (Plesiovelia) saulii Tamanini, 1947 is recorded for the first time Heteroptera fauna of Turkey.

Keywords: Heteroptera, fauna, Ilgaz Mountains, Kastamonu, Çankırı, Turkey

\section{Ilgaz Dağları (Kastamonu-Çankırı) Heteroptera Faunası ve Türkiye İçin İlave Yeni Kayit}

Öz

Bu araştırma Mayıs 1999 ve Eylül 2000 arasında Ilgaz Dağlarında yürütülmüş olup Heteroptera takımının 16 familyasının (Notonectidae, Gerridae, Veliidae, Miridae, Anthocoridae, Reduviidae, Nabidae, Berytidae, Lygaeidae, Pyrrhocoridae, Stenocephalidae, Coreidae, Alydidae, Rhopalidae, Scutelleridae, Pentatomidae) 73 cinsine dahil 93 tür ve alttür tespit edilmiştir. Belirlenen 93 taksonun 75’i Ilgaz Dağları, Velia (Plesiovelia) saulii Tamanini, 1947 de Türkiye Heteroptera faunası için ilk kez kaydedilmektedir.

Anahtar Kelimeler: Heteroptera, Fauna, Ilgaz Dağları, Kastamonu, Çankırı, Türkiye

* e-mail: basmacii@gmail.com 


\section{Introduction}

The Heteroptera is one of the largest and most diverse groups of insects. They are generally treated as a suborder of the Hemiptera. Heteroptera lives on all continents (except Antarctica) and on many islands [1]. The Heteroptera (true bugs) is a significant insect taxon with approximately 42.300 described species worldwide and it is separated into seven infraorders and 75-89 families [1 and 2]. The climate and vegetation diversity in Turkey and the factors such as vastness of geological location bring diversity to insects that including the Heteroptera order. The Heteroptera fauna in Turkey, especially in the $19^{\text {th }}$ and $20^{\text {th }}$ centuries, has been studied by both native and foreign researchers, thus significant findings were obtained [3]. According to Henry [2], there are 1.518 genera and 8.350 species in the Palearctic region. Önder et al. [3] determined 1.526 Heteroptera species/subspecies belonging to 40 different families in Turkey by the end of year 2006.

Ilgaz Mountains is a transition region between Central Anatolia and Black Sea region of Turkey. Therefore, in this transition area is abundant variety of plants. Hence the diversity and abundance of insects is more. According to initial records, 82 Heteropteran species were collected from Ilgaz Mountains [4-11].

The aim of this study is to determine Heteroptera fauna of Ilgaz Mountains (Kastamonu, Çankırı) and to make a contribution to Turkish Heteroptera fauna.

\section{Material and Methods}

This study is based on 474 specimens of Heteroptera and all the specimens were collected by the first author from Ilgaz Mountains (Kastamonu, Çankırı) between May 1999 and September 2000 for the Master of Sciences thesis [12].

Samples were collected by beating the vegetation with hand-net. Specimens were killed in jars that were filled with ethyl acetate, prepared and labeled according to the standard procedures for museum material. Coordinates of species "Microsoft Office Excel" program and transferred to the "ESRI ArcView GIS version 3.1" map program, on the map localities of the species caught were processed by treatmen. A list of localities is given in Figure 1. The beetles were identified using the stereomicroscope. For determining the species of examples, the diagnostic keys and descriptions in the related literatures were used [10, 13-22].

The material examined under headings; males and females number of captured species, locality data, GPS coordinates, altitude and captured time is given for each species. Materials have been deposited in Zoological Museum, Kastamonu University, Turkey. 


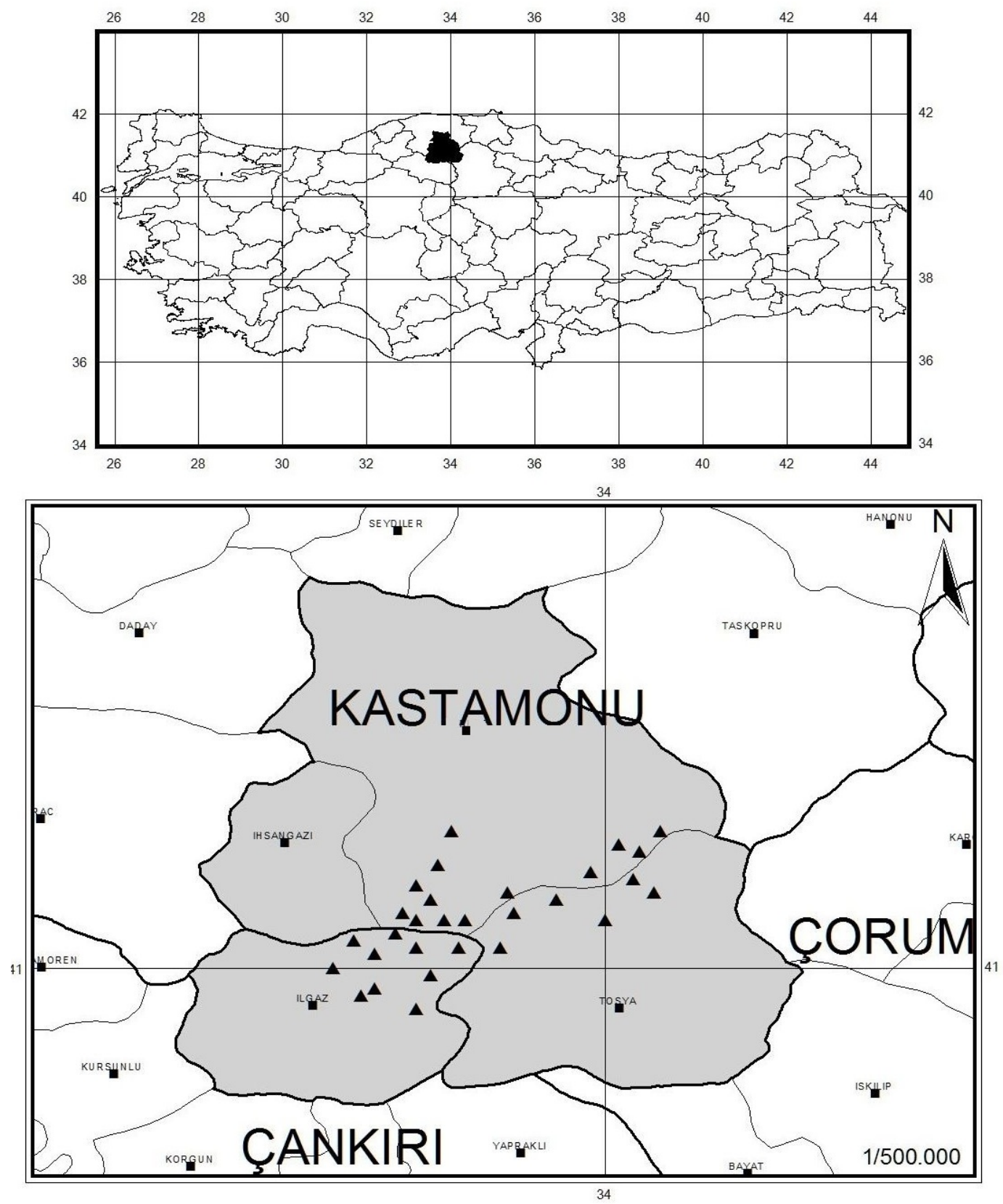

Figure 1. Ilgaz Mountains study area and collected localities $(\boldsymbol{\Lambda})$

\section{Results}

At the end of this study, 93 species and subspecies which belong to sixteen families of Heteroptera have been determined. The following distributions of these species are given separately for each species. Villages in the text abbreviated as “v.”.

\section{Heteroptera}

Family: Notonectidae Latreille, 1802

\section{Notonecta marmorea Fabricius, 1803}

Materials examined: 4 specimen, Kastamonu, Tosya, (Dipsiz Lake), 4100’55.64”N/3352’34.65”E, 1478m, 12.VII.2000; 2 specimen, Kastamonu, Centrum, (Ilgaz Mountain Ski Center), 
4104’40.67’N/3343’45.54”E, 1800m, 05.VIII.2000; 8 specimen, Kastamonu, Centrum, (Baldiran), 4105’01.67’N/3345’02.34”E, 1546m, 13.VIII.2000; 10 specimen, Çankırı, Ilgaz, (Kırkpınar Plateau), 4100’19.36”'N/33³7’14.09”'E, 1800m, 17.IX.2000.

Distribution in the world: Albania, Corsica, Cyprus, Former Yugoslavia, Greece, Iran, Italy, Poland, Sardinia, Sicily, South Russia, Syria, Turkey, Turkistan, Turkmenistan [18].

\section{Family: Gerridae Leach, 1815}

2. Gerris (s. str.) costae costae (Herrich-Schäffer, 1850)

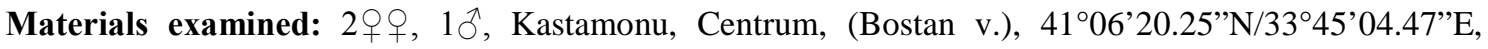

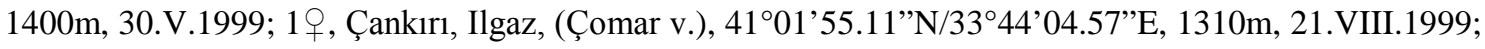
1q, Kastamonu, Centrum, (Çatören v.), 4108’33.89”N/3346’55.82”E, 1200m, 18.IX.1999; 2 우, Kastamonu, Centrum, (East of Ilgaz Mountain Pass), 4103’54.67’N/3345’08.79”E, 1875m, 11.VI.2000;

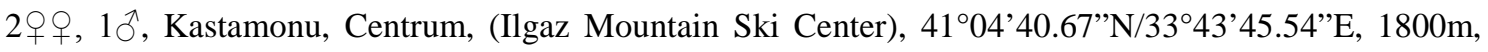

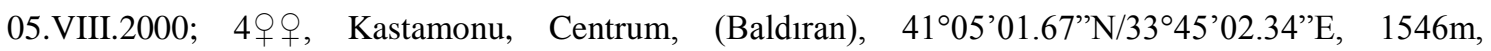
13.VIII.2000; 1ㅇ, 1§̂, Kastamonu, Centrum, (Gökçukur v.), 41¹6’47.54”N/3355’09.88”E, 1250m, 03.IX.2000.

Distribution in the world: Palestine, South West Asia, Syria, Transcaucasia, Turkey [6].

\section{Gerris (Gerriselloides) lateralis Schummel, 1832}

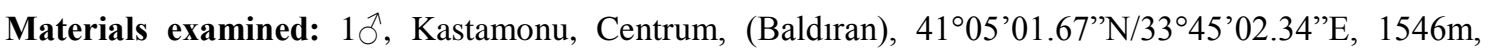
13.VIII.2000.

Distribution in the world: Austria, Belarus, Belgium, Central Russia, Denmark, East Russia, Estonia, Finland, Former Yugoslavia, Germany, Iran, Iraq, Ireland, Italy, Latvia, Liechtenstein, Lithuania, Macedonia, Netherlands, North Russia, Norway, Poland, Portugal, Romania, Slovakia, South Russia, Spain, Sweden, Switzerland,Transcaucasia, Turkey, Ukraine, United Kingdom [6].

\section{Aquarius najas (De Geer, 1773)}

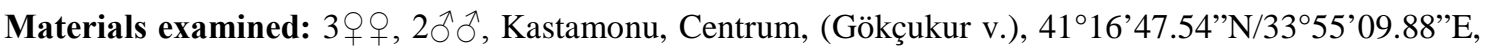
1250m, 03.IX.2000.

Distribution in the world: Albania, Austria, Baltic States, Belgium, Corsica, Czech Republic, Denmark, England, Finland, Former Yugoslavia, France, Germany, Hungary, Ireland, Italy, Morocco, Netherlands, Norway, Poland, Portugal, Sardinia, Scotland, Sicily, South Russia,Spain, Sweden, Switzerland, Syria, Western Russia [18].

Family: Veliidae Amyot \& Serville, 1843

\section{Velia (Plesiovelia) saulii Tamanini, 1947}

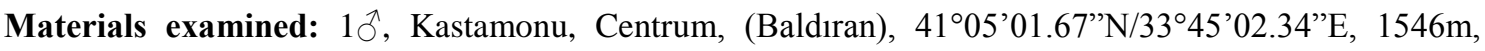
13.VIII.2000.

Distribution in the world: Austria, Belgium, Croatia, Czech Republic, Denmark, England, Estonia, Finland, Former Yugoslavia,France, Hungary, Ireland, Italy, Liechtenstein, Lithuania, Netherlands, Norway, Poland, Portugal, Romania, Russia, Slovakia, Slovenia, Spain, Sweden, Switzerland, Ukraine [23]. 
Remarks: Examples of species was found to the banks of the pond in the stream in the forest formation of Abies nordmanniana ssp. bornmuelleriana-Pinus sylvestris, $1546 \mathrm{~m}$. high. This species is new to fauna of Heteroptera of Turkey.

Family: Miridae Hahn, 1831

6. Fulvius punctumalbum (Rossi, 1790)

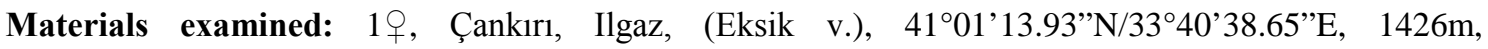

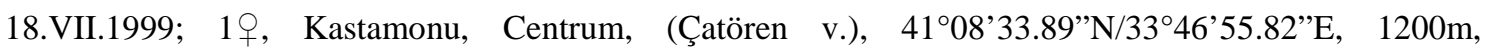
06.VIII.1999; 2qq, 1§, Kastamonu, Centrum, (Ilgaz Mountain Ski Center),

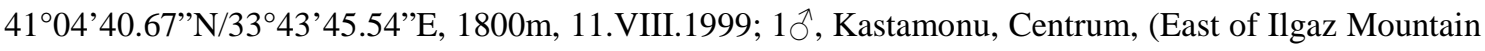
Pass), 4103’54.67’N/3345’08.79”E, 1875m, 13.VIII.2000.

Distribution in the world: Albania, Crimea, Former Yugoslavia, Greece, Italy, Romania, South France, South Russia, Spain, Transcaucasia, Turkey [14 and 18].

7. Bothynotus pilosus (Boheman, 1852)

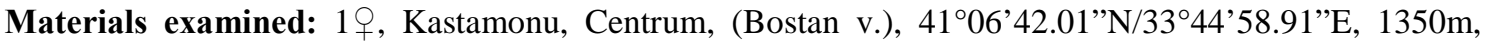

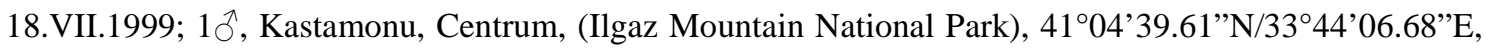
1750m, 18.VII.1999; 2 우, 06.VIII.1999; 1엉. Kastamonu, Centrum, (Ilgaz Mountain National Park Derbent), 4103’50.94’N/3344’38.36”E, 1800m, 06.VIII.2000.

Distribution in the world: Austria, Belgium, Czech Republic, Denmark, England, Finland, Former Yugoslavia, France, Germany, Greece, Hungary, Italy, Netherlands, Norway, Scotland, Sicily, South Russia, Sweden, Switzerland, Turkey,Western Russia [19 and 24].

8. Deraeocoris (s. str.) rutilus (Herrich-Schäffer, [1838])

Materials examined: 1, Çankırı, Ilgaz, (Eksik v.), 4101’13.93”N/3340’38.65”E, 1426m,

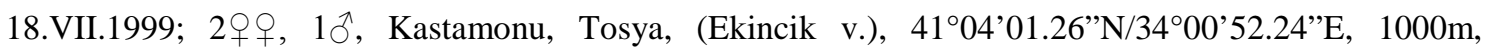
12.VII.2000; 1 ㅇ, Çankırı, Ilgaz, (Musaköy v.), 4058’04.05’N/3340’48.35’E, 1100m, 06.VIII.2000.

Distribution in the world: Albania, Bulgaria, Caucasus, Cyprus, Former Yugoslavia, Hungary, Israel, Italy, Poland, Romania, Sardinia, South Russia, Syria, Transcaucasia, Turkey [19, 25-29].

\section{Monalocoris (s. str.) filicis (Linnaeus, 1758)}

Materials examined: 19, Kastamonu, Tosya, (Güney v.), 41¹0’05.60”N/3403’54.17’E, 1250m, 07.VIII.1999.

Distribution in the world: Baltic States, Belgium, Canada, Czech Republic, Denmark, Finland, Former Yugoslavia, France, Italy, Kamchatka, Mexico, Netherlands, Norway, Poland, Romania, Siberia, Sicily, South Russia, Sweden, Turkey, USA, Western Russia [20 and 24].

\section{Megalocoleus tanaceti (Fallén, 1807)}

Materials examined: 1q, Çankırı, Ilgaz, (Eksik v.), 4101’13.93”N/3340’38.65”E, 1426m,

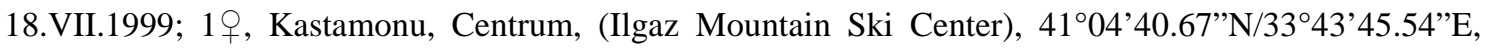
1800m, 05.VIII.2000.

Distribution in the world: Baltic States, Belgium, Czech Republic, Denmark, England, Finland, France, Germany, Hungary, Italy, Netherlands, Poland, Romania, Sardinia, Scotland, Siberia, South Russia, Sweden, Turkey,Western Russia [20 and 28].

\section{Plagiotylus dispar Reuter, 1899}


Materials examined: $2+\circ$, Kastamonu, Centrum, (East of Ilgaz Mountain Pass), 4103’54.67’N/3345’08.79’E, 1875m, 13.VIII.2000.

Distribution in the world: Israel, Turkey [22].

\section{Stenodema (s. str.) laevigatum (Linnaeus, 1758)}

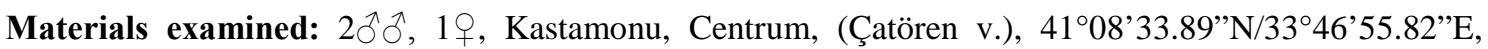
1200m, 06.VIII.1999; 2우, Kastamonu, Centrum, (Bostan v.), 4106’42.01”N/3344’58.91”E, 1350m, 18.VII.1999; 1우 Kastamonu, Centrum, (Bostan v.), 4106'20.25”N/3345’04.47’E, 1400m, 18.IX.1999; 3우우, Kastamonu, Centrum, (Ilgaz Mountain National Park), 4104’39.61’N/3344’06.68’E, 1750m, 18.VII.1999.

Distribution in the world: Afghanistan, Albania, Algeria, Austria, Baltic countries, Belgium, Bulgaria, Canada, Caucasus, China,Czech Republic, Denmark, England,Finland, Former Yugoslavia, France, Germany, Greece, Hungary, Ireland, Israel, Italy, Morocco, Netherlands, Norway, Poland, Portugal, Romania, Sardinia, Scotland, Sicily, South Russia, Spain, Sweden, Switzerland, Syria, Turkestan, Turkey, United States, Western Russia [11, 19, 24, 25, 30].

13. Notostira erratica (Linnaeus, 1758)

Materials examined: 1ㅇ, Kastamonu, Centrum, (Küçük Hacet Hill), 4104’51.82”N/3348’43.93”E, 2500m, 09.IX.2000.

Distribution in the world: Algeria, Belgium, Bulgaria, Caucasus, England, Finland, Former Yugoslavia,France, Germany, Hungary, Iran, Israel, Italy, Mongolia, Morocco, Netherlands, Poland, Portugal, Romania, Russia, Siberia, Spain, Sweden, Switzerland, Syria, Transcaucasia, Turkestan, Turkey $[11,25,31]$.

\section{Leptopterna dolabrata (Linnaeus, 1758)}

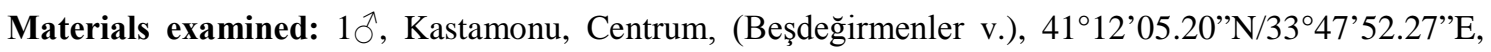

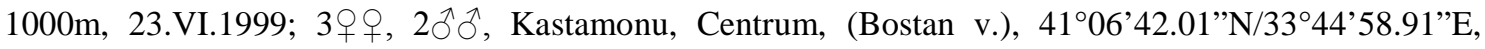
1350m, 18.VII.1999; 1q, 1ठ̂, Çankırı, Ilgaz, (Eksik v.), 4101’13.93”N/3340’38.65”E, 1426m,

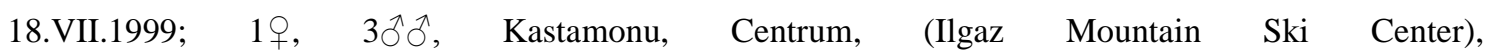

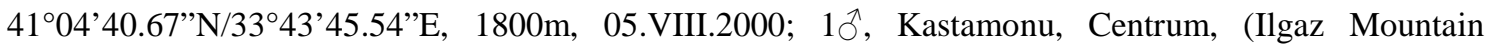
National Park), 4103’17.63”N/3343’07.27’E, 2000m, 13.VIII.2000.

Distribution in the world: Albania, Austria, Baltic States, Belgium, Bulgaria, Canada, Caucasus, Czech Republic, Denmark, England, Finland, Former Yugoslavia, France, Germany, Hungary, Italy, Kamchatka, Netherlands, Norway, Poland, Portugal, Romania, Russia, Scotland, Siberia, South Russia, Spain, Sweden, Switzerland, Tajikistan, Transcaucasia, Turkey, USA, Western Russia [11,19, 25].

\section{Adelphocoris vandalicus (Rossi, 1790)}

Materials examined: $2 \circ \bigcirc$, Kastamonu, Tosya, (Dipsiz Lake), 4100’55.64”N/3352’34.65”E, 1478m, 12.VII.2000.

Distribution in the world: Albania, Austria, Bulgaria, Caucasus, Corsica, Czech Republic, Former Yugoslavia, France, Germany, Hungary, Italy, Morocco, Poland, Romania, Sardinia, Sicily, South Russia, Spain, Switzerland, Transcaucasia, Turkestan, Western Russia [20 and 25].

16. Closterotomus reuteri (Horvath, 1882) 
Materials examined: 1 , Kastamonu, Centrum, (Ilgaz Mountain National Park), 4104’39.61’N/3344’06.68”E, 1750m, 18.VII.1999.

Distribution in the world: Former Yugoslavia, Turkey [10, 19, 25].

17. Grypocoris (Turciocoris) heinzi Wagner, 1966

Materials examined: 1 + $1 \hat{\jmath}$, Kastamonu, Centrum, (Ilgaz Mountain National Park),

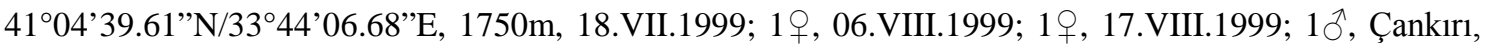

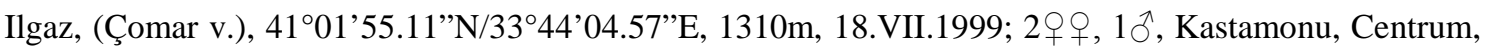
(East of Ilgaz Mountain Pass), 4103’54.67’N/3345’08.79’E, 1875m, 06.VIII.1999.

Distribution in the world: Turkey [10, 24, 30].

18. Aphanosoma italicum Costa, 1842

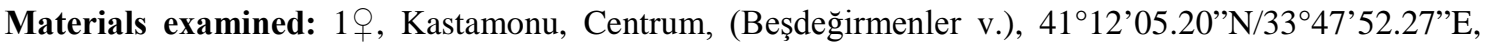
1000m, 23.VI.1999; 2 우, Kastamonu, Centrum, (Bostan v.), 4106’42.01”N/3344’58.91”E, 1350m, 18.VII.1999; 2우, Kastamonu, Centrum, (Bostan v.), 4106’24.81”N/3344’55.42”E, 1450m, 05.VIII.2000.

Distribution in the world: Austria, Bulgaria, Caucasus, Former Yugoslavia, Greece, Italy, Romania, Siberia, Southern Russia, Turkey [10, 16, 19, 25, 29, 30, 32].

\section{Brachycoleus decolor Reuter, 1887}

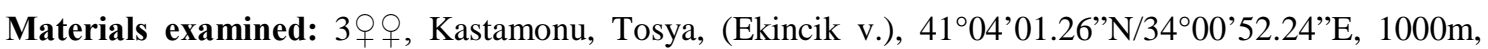
12.VII.2000.

Distribution in the world: Albania, Austria, Bulgaria, Czech Republic, Former Yugoslavia, France, Germany, Hungary, Romania, Sardinia, Siberia, Sicily, Spain, Switzerland, Turkestan, Turkey [19, 29, 31, 33].

\section{Stenotus binotatus (Fabricius, 1794)}

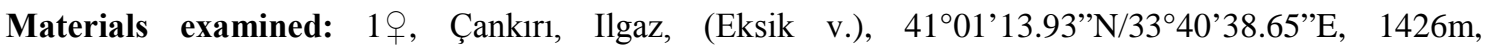

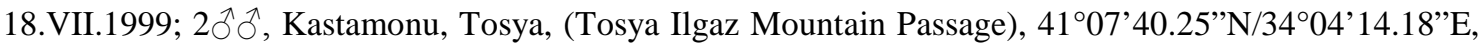

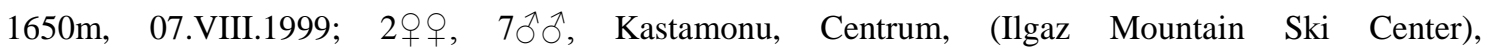
4104’40.67’N/3343’45.54”E, 1800m, 11.VIII.1999.

Distribution in the world: Albania, Austria, Baltic States, Bulgaria, Canada, Czech Republic, Denmark, England, Ethiopia, Finland, Former Yugoslavia, France, Germany, Greece, Hungary, Ireland, Italy, Netherlands, Norway, Poland, Portugal, Romania, Sicily, South Russia, Spain, Sweden, Switzerland, Turkey, USA, Western Russia [19, 25, 28, 30, 31].

\section{Liocoris tripustulatus (Fabricius, 1781)}

Materials examined: 19, Kastamonu, Centrum, (Çatören v.), 4108’33.89”N/3346’55.82”E, 1200m, 18.VII.1999; 2우, 2 specimen, 06.VIII.1999; 2qㅇ, Kastamonu, Centrum, (Bostan v.), 4106'24.81”N/3344'55.42”E, 1450m, 11.VIII.1999; 1, Kastamonu, Tosya, (Dipsiz Lake), 4100’55.64”N/3352’34.65”E, 1478m, 12.VII.2000; 1 specimen, Kastamonu, Centrum, (Bostan v.), 4106’20.25’N/3345’04.47’E, 1400m, 18.IX.1999; 1 specimen, Kastamonu, Centrum, (Ilgaz Mountain National Park), 4103’17.63”N/3343’07.27’E, 2000m, 13.VIII.2000.

Distribution in the world: Albania, Austria, Baltic States, Belgium, Bulgaria, Caucasus, Czech Republic, Denmark, England, Finland, Former Yugoslavia, France, Germany, Greece, Hungary, Ireland, 
Israel, Italy, Netherlands, Norway, Poland, Portugal, Romania, Sardinia, Scotland, Siberia, Sicily, South Russia, Spain, Syria, Transcaucasia, Turkestan, Turkey, Western Russia [15, 20, 25, 27, 29, 30].

22. Charagochilus (s. str.) gyllenhalii (Fallén, 1807)

Materials examined: 2 specimen, Kastamonu, Centrum, (East of Ilgaz Mountain Pass), 4103’54.67’N/3345’08.79”E, 1875m, 13.VIII.2000.

Distribution in the world: Algeria, Austria, Balkan Peninsula, Baltic States, Belgium, Bulgaria, Caucasus, China, Cyprus, Czech Republic, Denmark, England, Finland, Former Yugoslavia, France, Germany, Iran, Italy, Japan, Morocco, Netherlands, Norway, Poland, Portugal, Romania, Scotland, Siberia, Sicily, South Russia, Spain, Sweden, Switzerland, Syria, Tajikistan, Transcaucasia, Turkestan, Turkey, Western Russia [10, 19, 24, 25, 28].

Family: Anthocoridae Fieber, 1837

23. Anthocoris nemorum (Linnaeus, 1761)

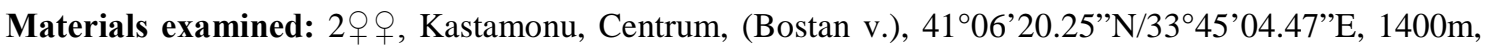

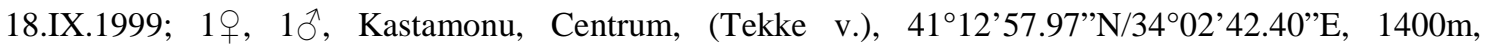
13.VI.2000.

Distribution in the world: Albania, Baltic States, Belgium, Bulgaria, China, Czech Republic, Denmark, Egypt, England, Finland, Former Yugoslavia, France, Germany, Hungary, Ireland, Italy, Kamchatka, Netherlands, Norway, Poland, Portugal, Romania, Scotland, Siberia, South Russia, Spain, Sweden, Switzerland, Turkey, Western Russia [20].

Family: Reduviidae Latreille, 1807

24. Rhynocoris (s. str.) punctiventris (Herrich-Schäffer, 1846)

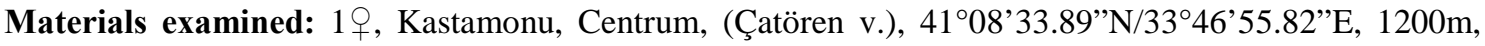

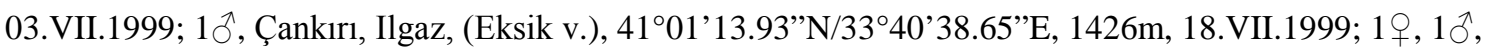
Çankırı, Ilgaz, (Onaç v.), 4058’48.05”N/3340’48.58”E, 1250m, 06.VIII.2000.

Distribution in the world: Albania, Bulgaria, Caucasus, Cyprus, Former Yugoslavia, South Russia, Syria, Transcaucasia, Turkey [21 and 25].

25. Sphedanolestes (s. str.) pulchellus (Klug, 1830)

Materials examined: $1 \hat{}$, Çankırı, Ilgaz, (Musaköy v.), 4058’04.05’N/3340’48.35”E, 1100m, 06.VIII.2000.

Distribution in the world: Albania, Cyprus, Greece, Israel, Syria, Turkey [21 and 25].

26. Nagusta goedeli (Kolenati, 1857)

Materials examined: $1 \delta^{\Uparrow}$, Çankırı, Ilgaz, (Musaköy v.), 4058’04.05”N/3340’48.35”E, 1100m, 06.VIII.2000.

Distribution in the world: Bulgaria, Caucasus, Cyprus, Czech Republic, Former Yugoslavia, Greece, Hungary, Iran, Iraq, Israel, Romania, Siberia, South Russia, Transcaucasia, Turkey [4 and 21].

Family: Nabidae Costa, 1852

27. Himacerus (s. str.) apterus (Fabricius, 1798)

Materials examined: $1 \delta$, Kastamonu, Centrum, (Çatören v.), 4108’33.89’N/3346’55.82”E, 1200m, 27.V.2000. 
Distribution in the world: Andorra, Austria, Belarus, Belgium, Bosnia and Herzegovina, Bulgaria, China, Croatia, Czech Republic, Denmark, England, Estonia, Former Yugoslavia, France, Germany, Greece, Hungary, Italy, Japan, Latvia, Liechtenstein, Lithuania, Luxembourg, Macedonia, Moldova, Netherlands, North Russia, Northwest Russia, Poland, Portugal, Romania, Siberia, Slovakia, Slovenia, South Russia, Spain, Switzerland, Turkey, Ukraine [15 and 21].

\section{Nabis (Tropiconabis) capsiformis Germar, 1838}

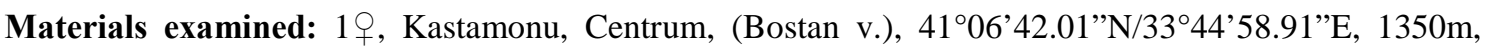
03.VII.1999; 1ㅇ, 18.VII.1999; 1ㅇ, Çankırı, Ilgaz, (Çomar v.), 4101’55.11”N/3344’04.57”E, 1310m,

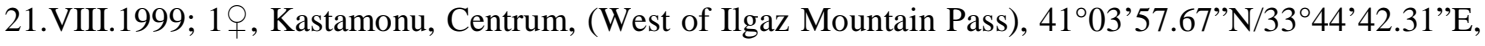

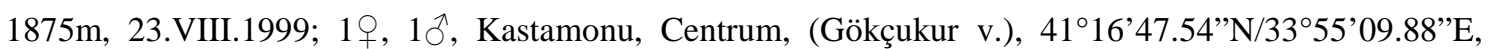
1250m, 12.VII.2000; 1ठิ, Kastamonu, Tosya, (Yukarı İsmailli v.), 4108’47.32”N/3404’10.01”E, 1400m, 07.VIII.1999.

Distribution in the world: Algeria, Australia, Austria, Bulgaria, Canary Islands, Cape Verde Islands, Caucasus, Ceylon, Corsica, Crimea, Cyprus, East Africa, Egypt, Ethiopia, Former Yugoslavia, Greece, Hungary, India, Iran, Italy, Japan, Libya, Madagascar, Malta, Morocco, Portugal, Sardinia, Sicily, South Russia, Southern France, Spain, The Azores, Tunisia, Turkestan, Turkey, Turkmenistan, Uzbekistan, Yemen [14, 21, 25, 30].

29. Nabis (s. str.) pseudoferus Remane, 1949

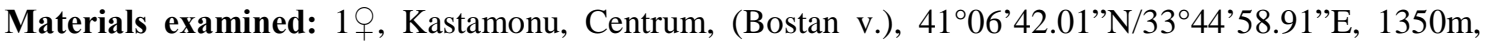
03.VII.1999; 1ㅇ, Kastamonu, Centrum, (Ilgaz Mountain Ski Center), 4104’40.67’N/3343’45.54”E, 1800m, 05.VIII.2000.

Distribution in the world: Austria, Bulgaria, Corsica, Cyprus, Czech Republic, England, Finland, Former Yugoslavia, France, Germany, Iran, Italy, Netherlands, Portugal, Sicily, Spain, The Azores, Turkey [20, 25, 28, 29].

\section{Nabis (Aspilaspis) viridulus Spinola, 1837}

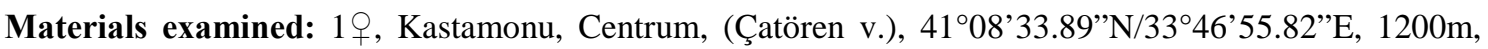

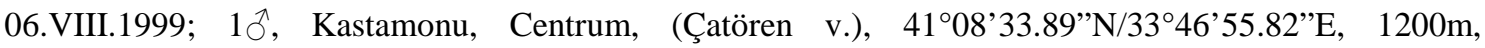
16.IX.2000; 1우 Kastamonu, Centrum, (West of Ilgaz Mountain Pass), 4103’57.67’N/3344’42.31”E, 1875m, 23.VIII.1999; 1q, Kastamonu, Centrum, (East of Ilgaz Mountain Pass), 4103’54.67’N/3345’08.79”E, 1875m, 13.VIII.2000.

Distribution in the world: Algeria, Canary Islands, Corsica, Cyprus, Egypt, Greece, Iran, Italy, Libya, Mongolia, Morocco, Portugal, Sardinia, Sicily, South Russia, Southern France, Spain, Syria, Tunisia, Turkestan, Turkey [21 and 25].

\section{Family: Berytidae Fieber, 1851}

\section{Neides tipularius (Linnaeus, 1758)}

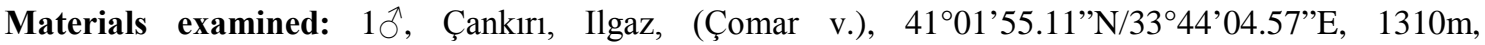

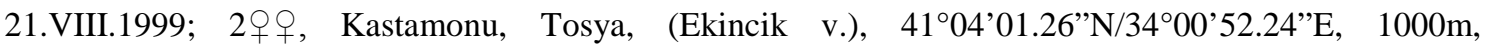
12.VII.2000.

Distribution in the world: Albania, Austria, Baltic countries, Belgium, Bulgaria, Caucasus, Crete, Czech Republic, Denmark, England, Finland, Former Yugoslavia, France, Germany, Greece, Hungary, Italy, 
Netherlands, Norway, Poland, Romania, Sicily, South Russia, Spain, Sweden, Switzerland, Transcaucasia, Turkestan, Turkey, Western Russia [20, 25, 30].

Family: Lygaeidae Schilling, 1829

32. Lygaeus equestris (Linnaeus, 1758)

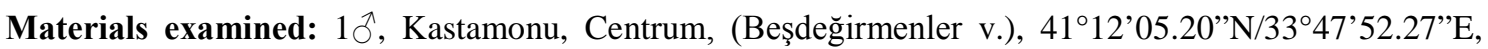
1000m, 23.VI.1999; 19, Kastamonu, Centrum, (Ilgaz Mountain National Park), 4104’39.61”N/3344’06.68”E, 1750m, 18.VII.1999; 19, Çankırı, Ilgaz, (Eksik v.),

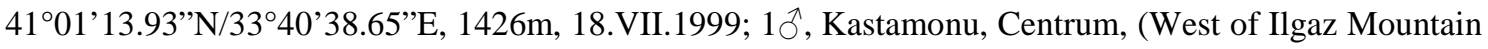
Pass), 4103’57.67”N/3344’42.31”E, 1875m, 23.VIII.1999; 1우 Kastamonu, Centrum, (Çatören v.), 4108’33.89”N/3346’55.82”'E, 1200m, 18.IX.1999.

Distribution in the world: Albania, Algeria, Baltic Countries, Caucasus, Central Europe, China, Corsica, Crete, Crimea, Cyprus, Denmark, Egypt, Finland, India, Iran, Iraq, Israel, Italy, Japan, Morocco, Norway, Poland, Portugal, Sardinia, Siberia, Sicily, Southern Europe, Spain, Sweden, Syria, Transcaucasia, Tunisia, Turkestan, Turkey, Western Russia [14, 15, 20, 25-31, 33, 34].

\section{Spilostethus saxatilis (Scopoli, 1763)}

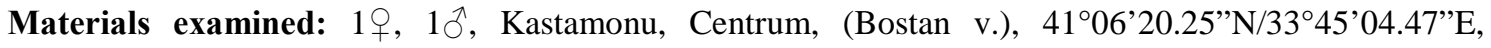
1400m, 06.VI.1999.

Distribution in the world: Afghanistan, Albania, Algeria, Andorra, Austria, Balearic Islands, Belgium, Bosnia and Herzegovina, Bulgaria, Canary Islands, Caucasus, Corfu Island, Corsica, Crete, Croatia, Cyprus, Czech Republic, Egypt, Former Yugoslavia, France, Germany, Greece, Hungary, India, Iran, Iraq, Israel, Italy, Kashmir, Lebanon, Liechtenstein, Luxembourg, Macedonia, Morocco, Netherlands, Poland, Portugal, Romania, Sardinia, Sicily, Slovakia, Slovenia, Spain, Switzerland, Syria, Tunisia, Turkestan, Turkey, Ukraine [20, 25, 27, 29, 30, 34].

\section{Melanocoryphus albomaculatus (Goeze, 1778)}

Materials examined: 1q, Çankırı, Ilgaz, (Onaç v.), 4058’48.05”N/3340’48.58”E, 1250m, 06.VIII.2000.

Distribution in the world: Albania, Belgium, Corsica, Czech Republic, Former Yugoslavia, France, Germany, Greece, Hungary, Italy, Libya, Portugal, Romania, Sardinia, South Russia, Spain, Sweden, Switzerland, Turkestan, Turkey [15, 20, 34].

\section{Ortholomus punctipennis (Herrich-Schäffer, 1838)}

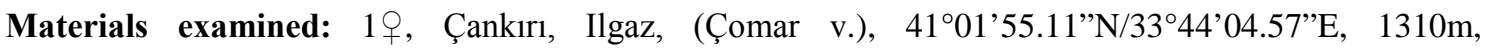
21.VIII.1999.

Distribution in the world: Alaska, Algeria, Austria, Balearic Islands, Baltic States, Belgium, Bulgaria,

Caucasus, China, Cyprus, Czech Republic, Denmark, England, Finland, Former Yugoslavia, France, Germany, Greece, Hungary, Italy, Mongolia, Netherlands, Norway, Poland, Romania, Siberia, Sicily, South Russia, Spain, Sweden, Switzerland, Transcaucasia, Turkestan, Turkestan, Western Russia [20, 25, 34].

36. Heterogaster affinis Herrich-Schäffer, 1835

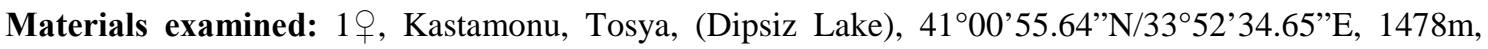

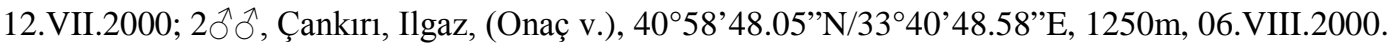


Distribution in the world: Albania, Algeria, Austria, Caucasus, Czech Republic, Former Yugoslavia, Germany, Greece, Hungary, Italy, Morocco, Portugal, Sicily, South Russia, Southern France, Switzerland, Transcaucasia, Turkestan, Turkey [20, 25, 27].

\section{Macroplax fasciata fasciata (Herrich-Schäffer, 1835)}

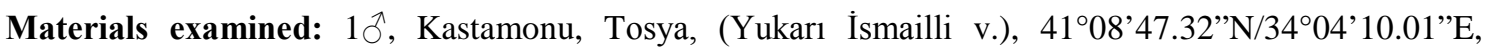
1400m, 07.VIII.1999.

Distribution in the world: Albania, Algeria, Austria, Balearic Islands, Bulgaria, Caucasus, Corfu Island, Corsica, Crete, Crimea, Cyprus, Czech Republic, Former Yugoslavia, France, Germany, Greece, Hungary, Iran, Iraq, Israel, Italy, Lebanon, Libya, Majorca, Morocco, Portugal, Romania, Sahara, Sardinia, Sicily, South Russia, Spain, Switzerland, Syria, Transcaucasia, Tunisia, Turkey, Turkmenistan [20, 24, 25, 29, 31, 34].

\section{Gastrodes grossipes (De Geer, 1773)}

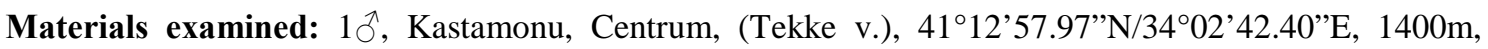
13.VI.2000.

Distribution in the world: Austria, Baltic countries, Belgium, Bulgaria, Corsica, Czech Republic, Denmark, Finland, Former Yugoslavia, France, Germany, Hungary, Ireland, Italy, Netherlands, Norway, Portugal, Scotland, Siberia, Sicily, South Russia, Spain, Sweden, Switzerland, Turkey, Western Russia [20].

\section{Emblethis griseus (Wolff, 1802)}

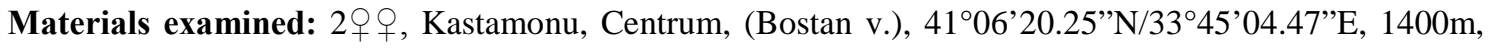
05.VI.1999; 1ㅇ, Çankırı, Ilgaz, (Eksik v.), 4101’13.93”N/3340’38.65”E, 1426m, 11.VI.2000.

Distribution in the world: Albania, Algeria, Austria, Bulgaria, Canary Islands, Caucasus, Cyprus, Czech Republic, Egypt, Former Yugoslavia, France, Germany, Greece, Hungary, Israel, Italy, Netherlands, Poland, Portugal, Romania, Siberia, Sicily, Spain, Switzerland, Syria, Transcaucasia, Tunisia, Turkestan, Turkey,United States, Western Russia [15, 20, 24, 25].

\section{Peritrechus geniculatus (Hahn, 1832)}

Materials examined: $1 \hat{\jmath}$, Kastamonu, Tosya, (Tosya Ilgaz Mountain Passage), 4107’40.25”N/3404’14.18”'E, 1650m, 03.IX.2000.

Distribution in the world: Algeria, Austria, Belgium, Bulgaria, Caucasus, Czech Republic, Denmark, England, Finland, France, Germany, Hungary, Iran, Italy, Netherlands, Norway, Poland, Portugal, South Russia, Spain, Sweden, Switzerland, Transcaucasia, Tunisia, Turkey, Western Russia [20, 25, 27].

\section{Peritrechus gracilicornis Puton, 1887}

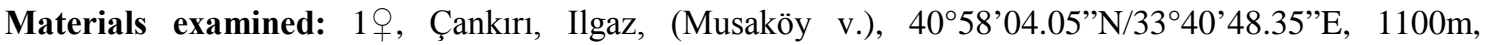
06.VIII.2000.

Distribution in the world: Albania, Algeria, Austria, Belgium, Bulgaria, Caucasus, Czech Republic, Denmark, Egypt, England, Former Yugoslavia, France, Germany, Greece, Hungary, Iran, Italy, Morocco, Netherlands, Poland, Portugal, Romania, Sardinia, Sicily, South Russia, Spain, Switzerland, Tajikistan, Transcaucasia, Tunisia, Turkestan, Turkey [20, 24, 25].

\section{Rhyparochromus pini (Linnaeus, 1758)}




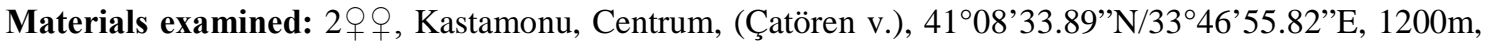

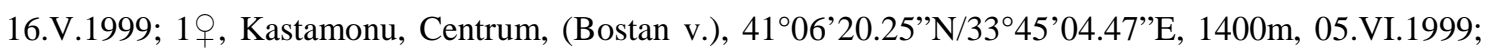
1q, Kastamonu, Centrum, (Bostan v.), 4106’20.25”N/3345’04.47’E, 1400m, 18.IX.1999; 19, Kastamonu, Centrum, (Ilgaz Mountain National Park Derbent), 4103’50.94’N/3344’38.36”E, 1800m,

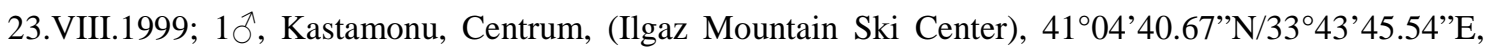

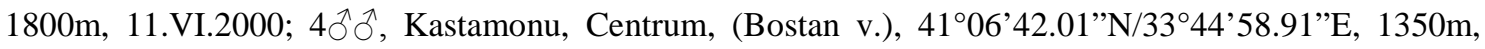
20.VIII.2000.

Distribution in the world: Albania, Baltic States, Belgium, Bulgaria, Caucasus, Czech Republic, Denmark, England, Finland, Former Yugoslavia, France, Germany, Greece, Hungary, Iran, Italy, Mongolia, Netherlands, Norway, Poland, Portugal, Romania, Siberia, Sicily, South Russia, Spain, Sweden, Switzerland, Tibet, Transcaucasia, Turkestan, Turkey, Western Russia [20 and 25].

\section{Beosus maritimus (Scopoli, 1763)}

Materials examined: $1 \sigma^{\jmath}$, Kastamonu, Centrum, (Çatören v.), 4108’33.89”N/3346’55.82”E, 1200m, 27.V.2000.

Distribution in the world: Algeria, Austria, Belgium, Canary Islands, Caucasus, Czech Republic, England, France, Germany, Iran, Iraq, Israel, Italy, Morocco, Netherlands, Poland, Portugal, Sardinia, Sicily, South Russia, Spain, Transcaucasia, Tunisia, Turkmenistan [15, 20, 25, 31].

Family: Pyrrhocoridae Fieber, 1860

44. Pyrrhocoris apterus (Linnaeus, 1758)

Materials examined: 1q, Çankırı, Ilgaz, (Kale v.), 4057’43.99’N/33³9’07.87’E, 1150m, 17.IX.2000;

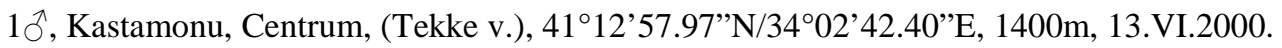

Distribution in the world: Albania, Andorra, Austria, Belarus, Belgium, Bosnia and Herzegovina, Bulgaria, Croatia, Cyprus, Czech Republic, Denmark, East Russia, England, Estonia, Former Yugoslavia, France, Germany, Greece, Hungary, Italy, Latvia, Liechtenstein, Lithuania, Luxembourg, Macedonia, Moldova, Netherlands, North Africa, North Russia, Northwest Russia, Poland, Portugal, Romania, Slovakia, Slovenia, South Russia, Spain, Sweden, Switzerland, The Middle East Asia, Turkey, Ukraine $[13,15,20,25,29,30]$.

\section{Family: Stenocephalidae Dallas, 1852}

45. Dicranocephalus agilis (Scopoli, 1763)

Materials examined: 1, Çankırı, Ilgaz, (Eksik v.), 4101’13.93”N/3340’38.65”E, 1426m,

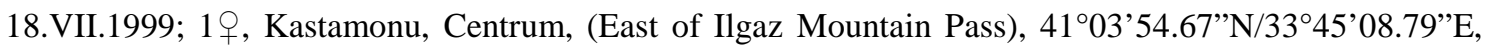
1875m, 13.VIII.2000.

Distribution in the world: Albania, Algeria, Austria, Balearic Islands, Baltic States, Belgium, Bulgaria, Canary Islands, Caucasus, Corsica, Crete, Cyprus, Czech Republic, England, Former Yugoslavia, France, Germany, Greece, Hungary, Iran, Italy, Morocco, Netherlands, Poland, Portugal, Romania, Sardinia, Scotland, Siberia, Sicily, South Russia, Spain, Switzerland, Transcaucasia, Tunisia, Turkey, Western Russia [15, 17, 20, 25].

\section{Dicranocephalus albipes (Fabricius, 1781)}

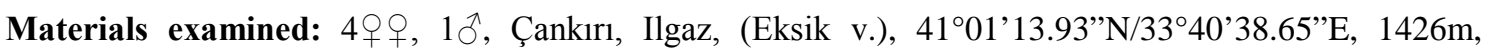
18.VII.1999; 1ð̊, Kastamonu, Tosya, (Güney v.), 41¹0’05.60”N/3403’54.17’E, 1250m, 07.VIII.1999. 
Distribution in the world: Albania, Austria, Azores, Bosnia and Herzegovina, Bulgaria, Caucasus, Croatia, Cyprus, Czech Republic, Former Yugoslavia, France, Germany, Greece, Hungary, Iran, Iraq, Israel, Italy, Macedonia, Madeira, Malta, Moldova, North Africa, North Russia, Northwest Russia, Poland, Portugal, Romania, Slovakia, Slovenia, South Russia, Spain, Switzerland, Syria, Transcaucasia,

Turkey, Ukraine [17, 20, 25, 29, 30].

\section{Family: Coreidae Leach, 1815}

\section{Gonocerus acuteangulatus (Goeze, 1778)}

Materials examined: 1 우 $1 \hat{\jmath}$, Kastamonu, Tosya, (Tosya Ilgaz Mountain Passage),

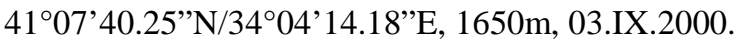

Distribution in the world: Albania, Austria, Belgium, Bulgaria, Caucasus, Central Asia countries, Central Europe, Cyprus, Czech Republic, England, Former Yugoslavia, France, Germany, Greece, Hungary, Iran, Italy, Netherlands, Poland, Portugal, Romania, Sardinia, Sicily, South Russia, Spain, Switzerland, Transcaucasia, Turkey, Turkmenistan, Western Russia [20 and 25].

\section{Gonocerus juniperi Herrich-Schäffer, 1839}

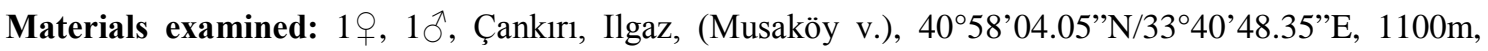
06.VIII.2000.

Distribution in the world: Austria, Bulgaria, Caucasus, Central Europe, Cyprus, Czech Republic, Former Yugoslavia, France, Germany, Greece, Hungary, Italy, Netherlands, Poland, South Russia, Switzerland, Transcaucasia, Turkey [20, 25, 29, 30].

\section{Coreus marginatus marginatus (Linnaeus, 1758)}

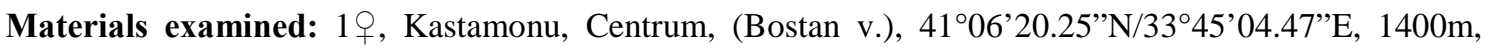

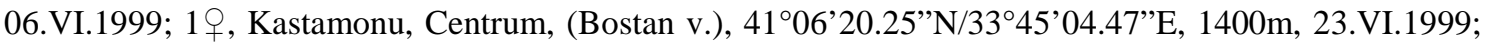

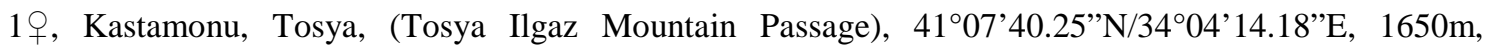

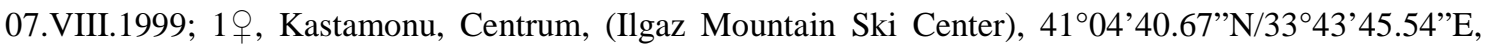

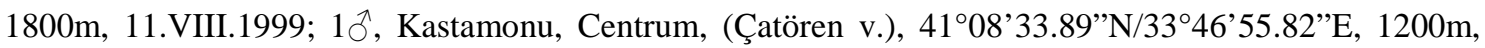

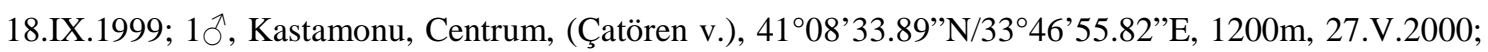

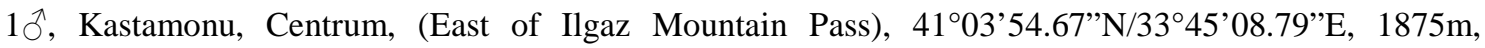

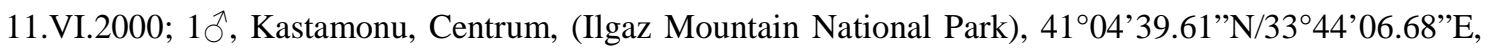
1750m, 20.VIII.2000.

Distribution in the world: Albania, Andorra, Austria, Belarus, Belgium, Bosnia and Herzegovina, Bulgaria, Caucasus, China, Crete, Croatia, Czech Republic, Denmark, England, Estonia, Finland, Former Yugoslavia, France, Germany, Greece, Hungary, Iran, Iraq, Ireland, Israel, Italy, Japan, Kyrgyzstan, Latvia, Liechtenstein, Lithuania, Luxembourg, Macedonia, Moldova, Netherlands, Norway, Poland, Portugal, Romania, Russia, Siberia, Slovakia, Slovenia, South Russia, Spain, Sweden, Switzerland, Syria, Tajikistan, Transcaucasia, Turkestan, Turkey, Ukraine [15, 20, 25, 26, 29, 30].

\section{Syromastus rhombeus (Linnaeus, 1767)}

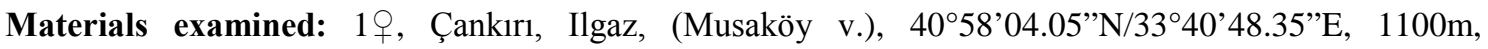

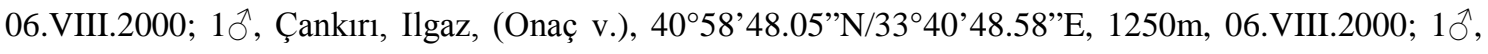

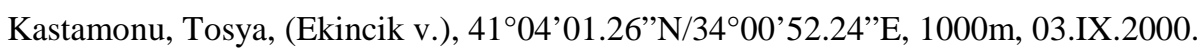


Distribution in the world: Albania, Algeria, Andorra, Austria, Belarus, Belgium, Bosnia and Herzegovina, Bulgaria, Canary Islands, Caucasus, Corsica, Croatia, Cyprus, Czech Republic, Denmark, England, Former Yugoslavia, France, Germany, Greece, Hungary, Iran, Iraq, Italy, Liechtenstein, Lithuania, Luxembourg, Macedonia, Madeira, Moldova, Morocco, Netherlands, North Russia, Northwest Russia, Poland, Portugal, Romania, Sardinia, Sicily, Slovakia, Slovenia, South Russia, Spain, Sweden, Switzerland, Syria, Transcaucasia, Turkestan, Turkey, Ukraine [15, 20, 25, 29].

\section{Enoplops scapha (Fabricius, 1794)}

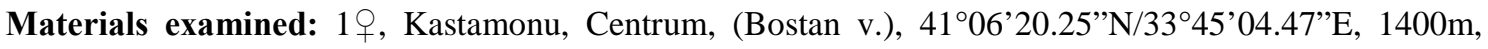
05.VI.1999.

Distribution in the world: Albania, Algeria, Austria, Baltic States, Belgium, Bulgaria, Caucasus, Czech Republic, England, Former Yugoslavia, France, Germany, Greece, Hungary, Israel, Italy, Netherlands, Poland, Portugal, Romania, Siberia, South Russia, Spain, Switzerland, Syria, Transcaucasia, Turkestan, Turkey, Western Russia [20 and 25].

\section{Ceraleptus gracilicornis (Herrich-Schäffer, 1835)}

Materials examined: 1 中 , Çankırı, Ilgaz, (Eksik v.), 4101'13.93”N/3340’38.65”'E, 1426m, 11.VI.2000. Distribution in the world: Albania, Algeria, Bulgaria, Caucasus, Czech Republic, Former Yugoslavia, Germany, Greece, Hungary, Iran, Italy, Morocco, Poland, Portugal, Romania, Southern France, Southern Russia, Spain, Switzerland, Transcaucasia, Turkey [20 and 25].

\section{Coriomeris hirticornis (Fabricius, 1794)}

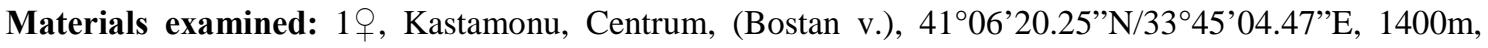
06.VI.1999; 1ð’, Kastamonu, Centrum, (Bostan v.), 4106’20.25”N/3345’04.47”E, 1400m, 23.VI.1999;

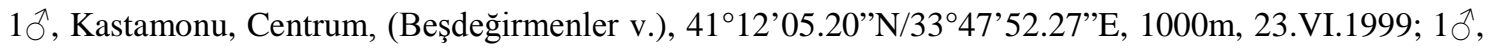

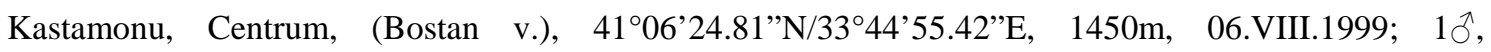
Kastamonu, Centrum, (Bostan v.), 4106’24.81”N/3344’55.42”E, 1450m, 11.VIII.1999; 19, Çankır1, Ilgaz, (Çomar v.), 4101’55.11”N/3344’04.57”E, 1310m, 21.VIII.1999; 1†, Çankırı, Ilgaz, (Eksik v.),

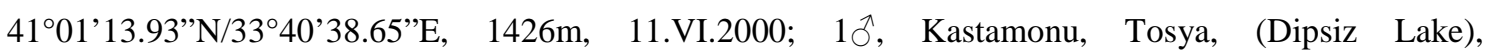

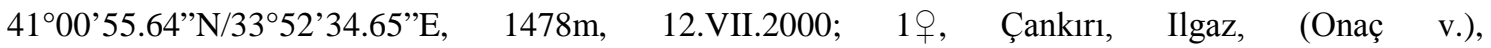

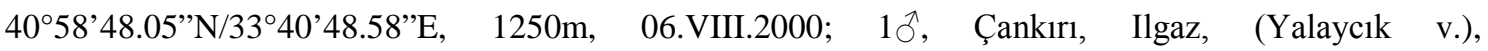

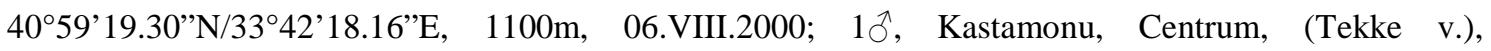
41¹2’57.97’N/3402’42.40”'E, 1400m, 13.VI.2000.

Distribution in the world: Albania, Algeria, Bulgaria, Caucasus, Central Europe, Corsica, Cyprus, Former Yugoslavia, Greece, Hungary, Israel, Italy, Lebanon, Morocco, Portugal, Romania, Sardinia, Sicily, South Russia, Southern France, Spain, Switzerland, Syria, Transcaucasia, Tunisia, Turkestan, Turkey [20, 25, 29].

\section{Family: Alydidae Amyot \& Serville, 1843}

\section{Alydus calcaratus (Linnaeus, 1758)}

Materials examined: 1, Kastamonu, Centrum, (Bostan v.), 4106’24.81”N/3344’55.42”E, 1450m, 11.VIII.1999; 2우, Kastamonu, Centrum, (Bostan v.), 4106’24.81”N/3344’55.42”E, 1450m, 17.VIII.1999; 1ㅇ, Çankırı, Ilgaz, (Musaköy v.), 4058’04.05”N/3340’48.35”E, 1100m, 06.VIII.2000;

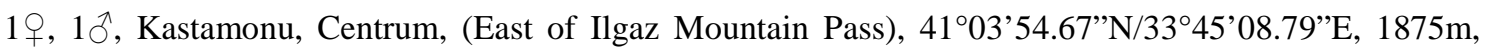


13.VIII.2000; 1ठ̂, Kastamonu, Centrum, (Bostan v.), 4106’42.01”N/3344’58.91”E, 1350m, 20.VIII.2000; 19, Kastamonu, Tosya, (Tosya Ilgaz Mountain Passage), 4107’40.25’N/3404’14.18’E, 1650m, 03.IX.2000.

Distribution in the world: Alaska, Albania, Algeria, Austria, Belarus, Belgium, Bosnia and Herzegovina, Bulgaria, Canada, Corsica, Croatia, Czech Republic, Denmark, England, Estonia, Finland, Former Yugoslavia, France, Germany, Greece, Hungary, Iran, Italy, Kamchatka, Latvia, Liechtenstein, Lithuania, Luxembourg, Macedonia, Moldova, Mongolia, Netherlands, North America, Norway, Poland, Portugal, Romania, Russia, Siberia, Slovakia, Slovenia, Spain, Sweden, Switzerland, Transcaucasia, Turkestan, Turkey, Ukraine, United States [17, 20, 25, 29, 30].

\section{Camptopus lateralis (Germar, 1817)}

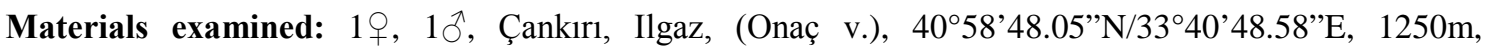

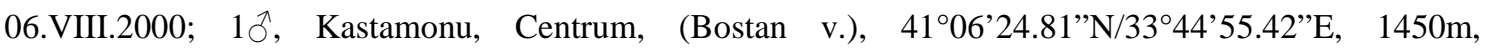

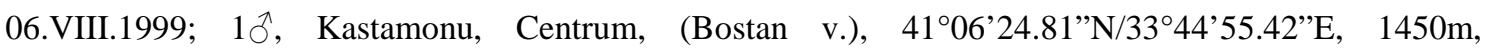

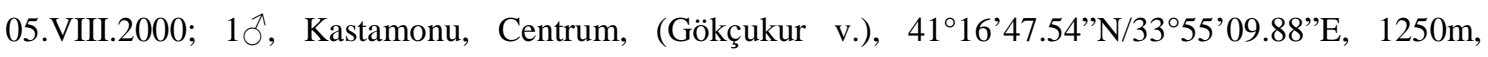

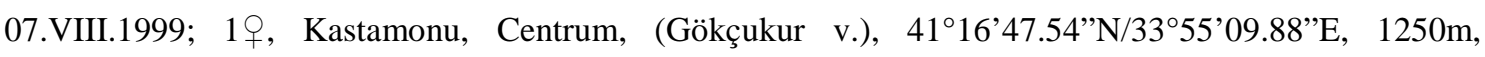
03.IX.2000; 1ð̄, Kastamonu, Centrum, (East of Ilgaz Mountain Pass), 4103’54.67’N/3345’08.79’E, 1875m, 13.VIII.2000.

Distribution in the world: Afghanistan, Caucasus, Central Europe, Cyprus, Czech Republic, Germany, India, Iran, Iraq, Israel, Kyrgyzstan, South Russia, Tajikistan, Transcaucasia, Turkestan, Turkey [15, 17, 20, 25, 29, 30, 33].

\section{Camptopus tragacanthae (Kolenati, 1845)}

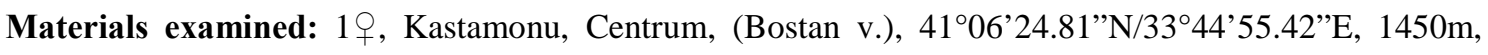
05.VIII.2000.

Distribution in the world: Caucasus, Iran, South Russia, Tajikistan, Transcaucasia, Turkey, Uzbekistan $[15,17,20,25,29]$.

\section{Family: Rhopalidae Amyot \& Serville, 1843}

57. Corizus hyoscyami hyoscyami (Linnaeus, 1758)

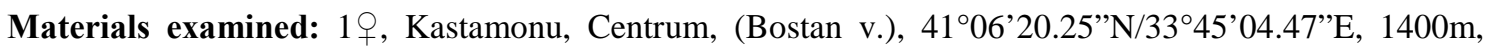
23.VI.1999; 19, Kastamonu, Centrum, (Bostan v.), 4106’42.01”N/3344’58.91”E, 1350m, 18.VII.1999;

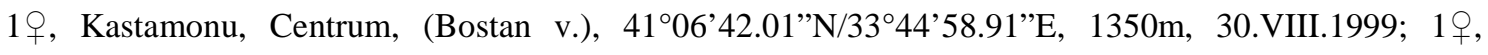
Kastamonu, Centrum, (Ilgaz Mountain National Park), 4104’39.61’N/3344’06.68”E, 1750m,

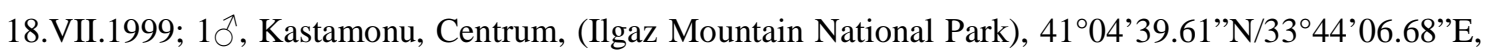
1750m, 06.VIII.1999; 19, Çankırı, Ilgaz, (Eksik v.), 4101’13.93”N/3340’38.65”E, 1426m,

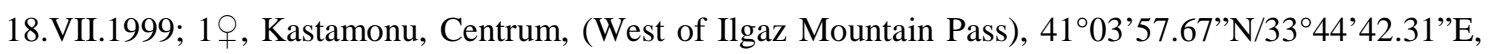
1875m, 06.VIII.1999; 1§̊, Kastamonu, Centrum, (Çatören v.), 4108’33.89’N/3346’55.82”E, 1200m, 06.VIII.1999.

Distribution in the world: Albania, Andorra, Austria, Belarus, Belgium, Bosnia and Herzegovina, Bulgaria, Caucasus, China, Crete, Croatia, Cyprus, Czech Republic, Denmark, England, Estonia, Finland, Former Yugoslavia, France, Germany, Greece, Hungary, Iran, Iraq, Ireland, Israel, Italy, Latvia, Liechtenstein, Lithuania, Luxembourg, Macedonia, Malta, Netherlands, North Africa, Norway, Poland, 
Portugal, Romania, Russia, Siberia, Slovenia, Spain, Sweden, Switzerland, Syria, Transcaucasia, Turkestan, Turkey, Turkmenistan, Ukraine [15, 17, 20, 25, 29, 30].

\section{Liorhyssus hyalinus (Fabricius, 1794)}

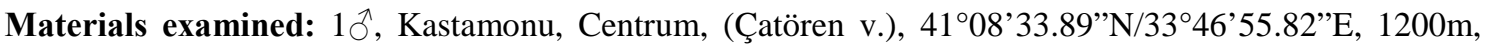

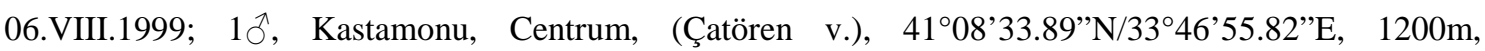

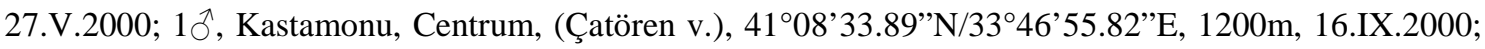
1오. Çankırı, Ilgaz, (Onaç v.), 4058’48.05”N/3340’48.58”E, 1250m, 06.VIII.2000.

Distribution in the world: Albania, Austria, Azores, Belarus, Belgium, Bosnia and Herzegovina, Bulgaria, Canary Islands, Caucasus, China, Croatia, Cyprus, Czech Republic, England, Finland, Former Yugoslavia, France, Germany, Greece, Hungary, Iran, Iraq, Ireland, Israel, Italy, Japan, Kyrgyzstan, Liechtenstein, Macedonia, Madeira, Malta, Moldova, Netherlands, North Africa, North Russia, Northwest Russia, Portugal, Romania, Siberia, Slovakia, Slovenia, South Russia, Spain, Sweden, Switzerland, Syria, Tajikistan, Transcaucasia, Turkestan, Turkey, Ukraine [15, 20, 25, 29].

\section{Rhopalus (s. str.) conspersus (Fieber, 1837)}

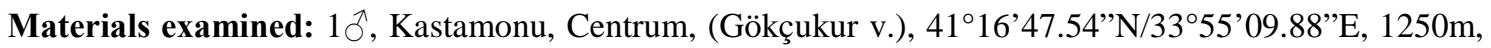
03.IX.2000

Distribution in the world: Austria, Bulgaria, Corsica, Czech Republic, Finland, Former Yugoslavia, France, Germany, Hungary, Iran, Italy, Morocco, Poland, Romania, Sardinia, South Russia, Spain, Switzerland, Turkestan, Turkey, Western Russia [17 and 20].

\section{Rhopalus (Aeschyntelus) maculatus (Fieber 1837)}

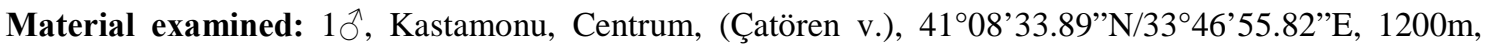

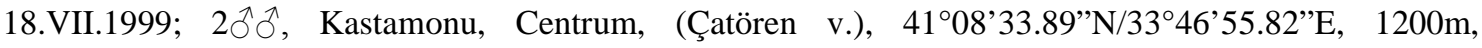
06.VIII.1999.

Distribution in the world: Albania, Algeria, Austria, Baltic States, Belgium, Bulgaria, Caucasus, Crete, Czech Republic, Denmark, England, Finland, Former Yugoslavia, France, Germany, Hungary, India, Iran, Iraq, Italy, Japan, Morocco, Netherlands, Norway, Poland, Portugal, Romania, Sardinia, Siberia, Sicily, South Russia, Spain, Sweden, Switzerland, Syria, Turkey, Western Russia [17, 20, 25].

\section{Rhopalus (s. str.) parumpunctatus Schilling, 1829}

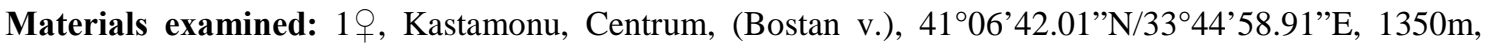

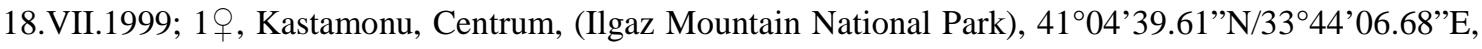
1750m, 18.VII.1999; 1ठ, Kastamonu, Centrum, (Ilgaz Mountain National Park),

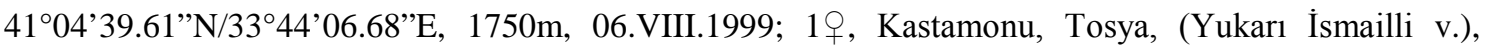
4108’47.32”N/3404’10.01”E, 1400m, 07.VIII.1999; 19, Kastamonu, Tosya, (Güney v.), 41¹0’05.60”N/3403’54.17”E， 1250m, 07.VIII.1999; 19, Çankır1, Ilgaz, (Çomar v.),

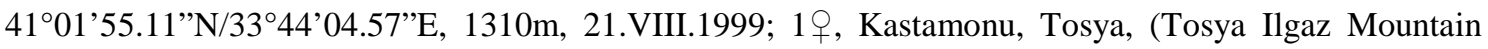

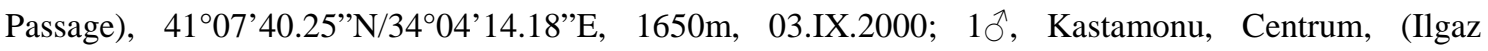
Mountain National Park), 4103’17.63”N/3343’07.27’E, 2000m, 13.VIII.2000; 1ठ, Kastamonu, Centrum, (Çatören v.), 4108’33.89’N/3346’55.82”E, 1200m, 06.VIII.1999; 10̋, Kastamonu, Centrum,

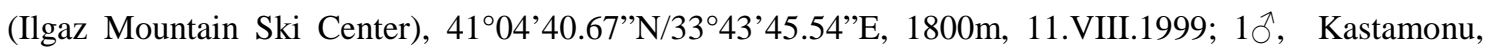
Tosya, (Ekincik v.), 4104’01.26”N/3400’52.24”E, 1000m, 03.IX.2000. 
Distribution in the world: Albania, Algeria, Austria, Baltic States, Belgium, Bulgaria, Canary Islands, Caucasus, Cyprus, Czech Republic, Denmark, England, Finland, Former Yugoslovia, France, Germany, Greece, Hungary, Iran, Italy, Kyrgyzstan, Morocco, Netherlands, Norway, Poland, Portugal, Romania, Sardinia, Siberia, Sicily, South Russia, Spain, Sweden, Switzerland, Syria, Tajikistan, Transcaucasia, Turkestan, Turkey, Western Russia [17, 20, 29, 30].

\section{Rhopalus (s. str.) rufus Schilling, 1829}

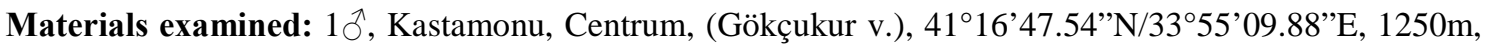
03.IX.2000.

Distribution in the world: Austria, France, Germany, Hungary, South Russia, Turkey [20 and 25].

\section{Stictopleurus crassicornis (Linnaeus, 1758)}

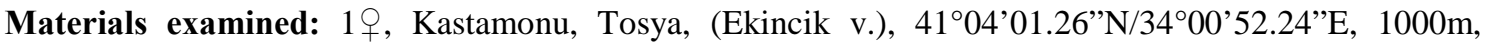
12.VII.2000; 1 , $10^{\Uparrow}$, Çankırı, Ilgaz, (Musaköy v.), 4058’04.05”N/3340’48.35”E, 1100m, 06.VIII.2000. Distribution in the world: Austria, Baltic countries, Belgium, Canary Islands, Caucasus, Czech Republic, Denmark, England, Finland, France, Germany, Hungary, Israel, Netherlands, Northern Italy, Norway, Poland, South Japan, South Russia, Sweden, Switzerland, Syria, Transcaucasia, Turkestan, Turkey, Western Russia [17, 20, 25, 30].

\section{Stictopleurus pictus (Fieber, 1861)}

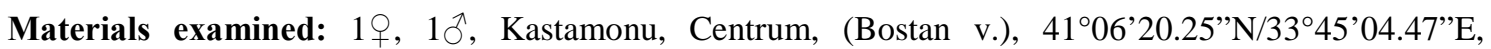

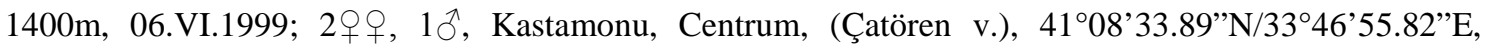

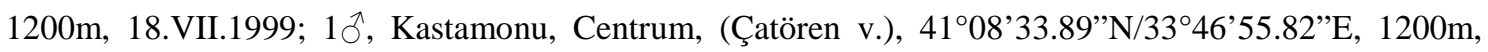

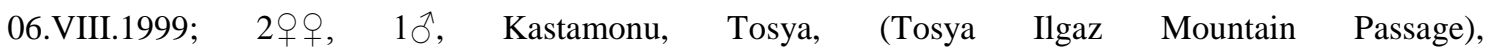

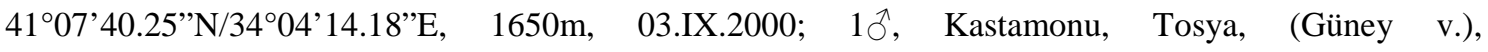

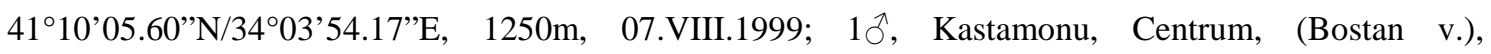

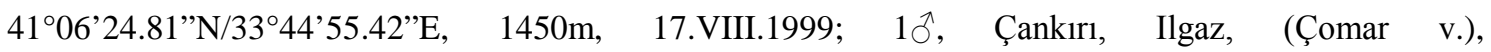

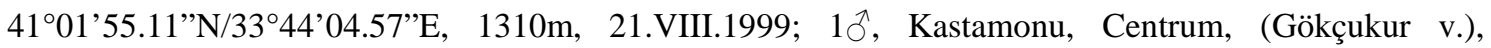

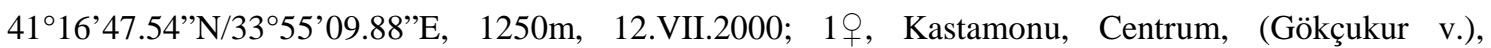
4116’47.54”N/3355’09.88”'E, 1250m, 03.IX.2000; 19, Kastamonu, Tosya, (Ekincik v.),

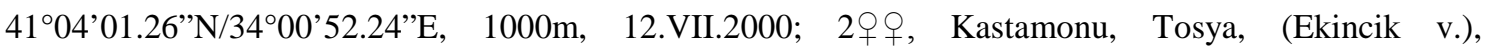

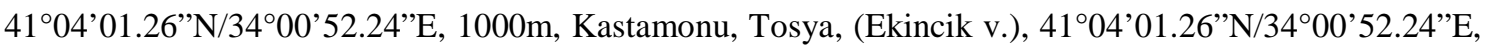

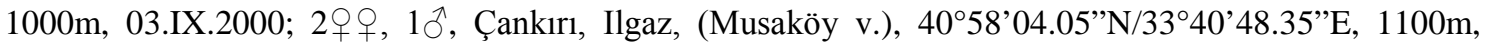

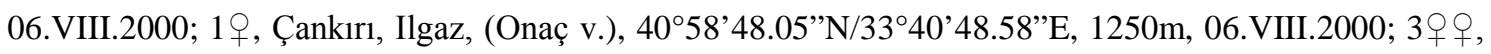

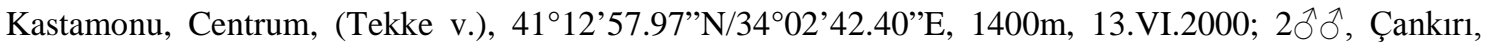

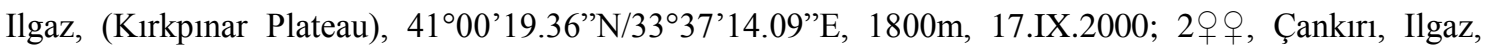
(Kale v.), 4057’43.99”N/33³9’07.87’'E, 1150m, 17.IX.2000.

Distribution in the world: Afghanistan, Canary Islands, Caucasus, Central Europe, Cyprus, England, Israel, Jordan, Mediterranean countries, North Africa, Southern Europe, Turkey, Ukraine [17].

\section{Maccevethus caucasicus (Kolenati, 1845)}

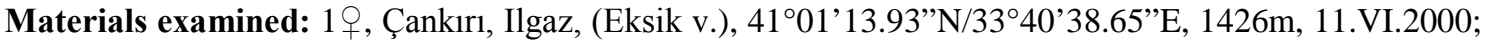
1ㅇ, Çankırı, Ilgaz, (Yalaycık v.), 4059’19.30”N/3342’18.16”E, 1100m, 06.VIII.2000. 
Distribution in the world: Albania, Algeria, Bulgaria, Central Europe, Corsica, Cyprus, Czech Republic, Former Yugoslavia, Greece, Hungary, Iran, Italy, Libya, Malta, Mediterranean countries, Morocco, North Africa, Portugal, Romania, Sardinia, Sicily, South Russia, Southern Europe, Southern France, Spain, Switzerland, Syria, Tunisia, Turkestan, Turkey, West Africa [17 and 20].

66. Maccevethus persicus Jakovlev, 1881

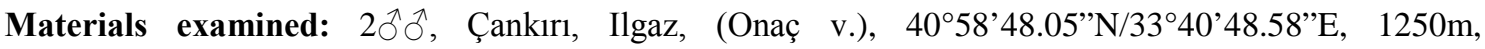
06.VIII.2000.

Distribution in the world: Bulgaria, Cape Verde Islands, Caucasus, Iran, Iraq, Sinai Peninsula, South Russia, Sudan, Syria, Transcaucasia, Turkestan, Turkey [15, 20, 25].

\section{Chorosoma schillingii (Schilling, 1829)}

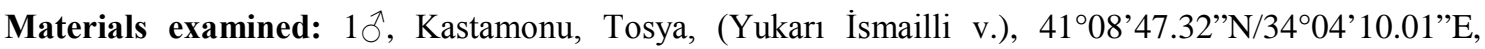
1400m, 07.VIII.1999; 1§, Kastamonu, Centrum, (Ilgaz Mountain National Park), 4104’39.61”N/3344’06.68”E, 1750m, 20.VIII.2000.

Distribution in the world: Albania, Austria, Belarus, Belgium, Bosnia and Herzegovina, Bulgaria, Caucasus, Croatia, Cyprus, Czech Republic, Denmark, England, Estonia, Finland, Former Yugoslavia, France, Germany, Greece, Hungary, Israel, Italy, Latvia, Lithuania, Macedonia, Moldova, Netherlands, North Africa, North Russia, Northwest Russia, Norwey, Poland, Portugal, Romania, Slovakia, South Russia, Spain, Sweden, Syria, Transcaucasia, Turkestan, Turkey, Ukraine, West Africa [17, 20, 25, 33].

\section{Family: Scutelleridae Leach, 1815}

68. Odontotarsus caudatus (Burmeister, 1835)

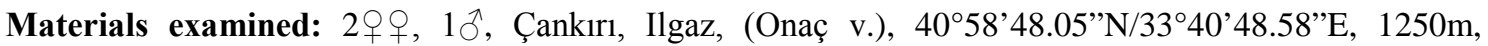
06.VIII.2000.

Distribution in the world: Albania, Balearic Islands, Canary Islands, Corsica, Egypt, Former Yugoslavia, Greece, Italy, Libya, Malta, Morocco, Portugal, Sardinia, Sicily, Spain, Turkey [20 and 24].

69. Odontotarsus robustus Jakovlev, [1884]

Materials examined: 1 ㅇ, Kastamonu, Centrum, (Bostan v.), 4106’20.25”N/3345’04.47”E, 1400m, 23.VI.1999; 1ð̋, Kastamonu, Tosya, (Güney v.), 41¹0’05.60”N/3403’54.17’E, 1250m, 07.VIII.1999;

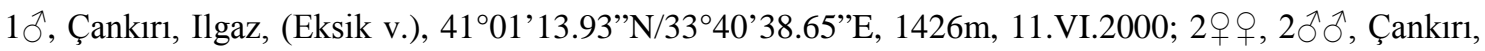
Ilgaz, (Onaç v.), 4058’48.05”N/3340’48.58”'E, 1250m, 06.VIII.2000; 1ð̄, Çankırı, Ilgaz, (Yalaycık v.), 4059’19.30”N/3342’18.16”E, 1100m, 06.VIII.2000.

Distribution in the world: Albania, Bulgaria, Caucasus, Corsica, Crete, Cyprus, Egypt, Former Yugoslavia, Greece, Hungary, Iran, Israel, Italy, Sicily, South Russia, Southern France, Spain, Syria, Turkestan, Turkey, Turkmenistan [20, 24, 25].

\section{Eurygaster hottentotta (Fabricius, 1775)}

Materials examined: 1q, Çankırı, Ilgaz, (Yalaycık v.), 4059’19.30”N/3342’18.16”'E, 1100m, 06.VIII.2000.

Distribution in the world: Algeria, Arabia, Azores, Bulgaria, Canary Islands, Greece, Italy, Libya, Morocco, Portugal, Sardinia, Sicily, South Russia, Southern France, Spain, Switzerland, Tunisia, Turkey [20 and 30].

71. Eurygaster maura (Linnaeus, 1758) 


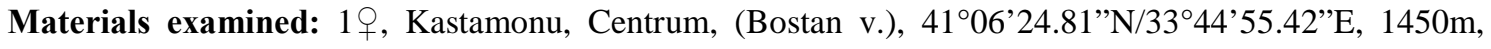

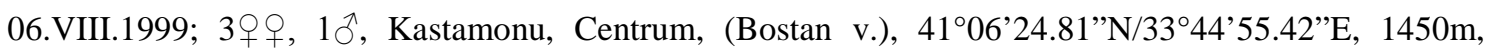

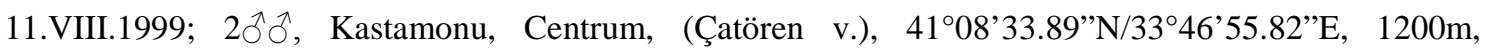
06.VIII.1999; 1ठ̃, Kastamonu, Centrum, (Ilgaz Mountain National Park Derbent),

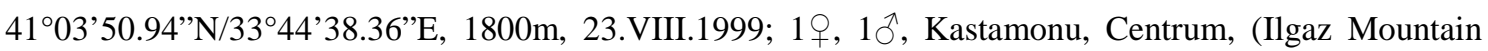

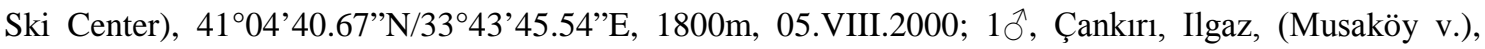

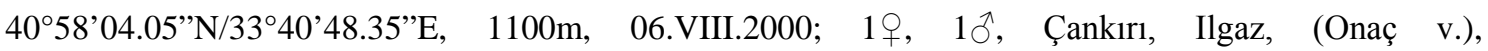
4058’48.05”N/3340’48.58”E, 1250m, 06.VIII.2000; 19, Kastamonu, Centrum, (Ilgaz Mountain

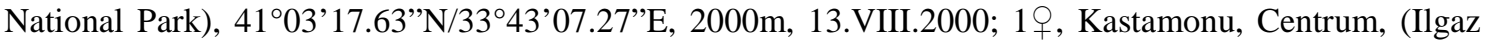
Mountain National Park), 4104’39.61’N/3344’06.68’E, 1750m, 20.VIII.2000.

Distribution in the world: Austria, Baltic countries, Belgium, Bulgaria, Caucasus, China, Corsica, Czech Republic, Denmark, England, Finland, Former Yugoslavia, France, Germany, Hungary, India, Ireland, Israel, Italy, Japan, Morocco, Netherlands, Norway, Poland, Portugal, Sardinia, Sicily, South Russia, Spain, Sweden, Switzerland, Syria, Transcaucasia, Turkestan, Turkey, Western Russia [20, 25-27, 29, 30, 31, 33].

\section{Eurygaster schreiberi Montandon, 1885}

Materials examined: 1 , Kastamonu, Centrum, (Ilgaz Mountain National Park), 4103’17.63”N/3343’07.27’E, 2000m, 13.VIII.2000.

Distribution in the world: Albania, Bulgaria, Former Yugoslavia, Italy, South France, South Russia, Turkestan, Turkey [20].

\section{Eurygaster testudinaria (Geoffroy, 1785)}

Materials examined: 1 ㅇ, Kastamonu, Centrum, (Beşdeğirmenler v.), 4112’05.20”N/3347’52.27’E,

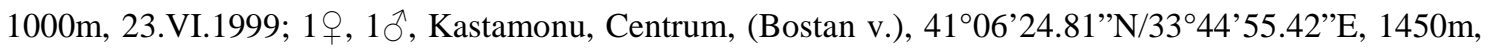

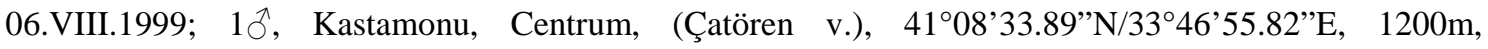

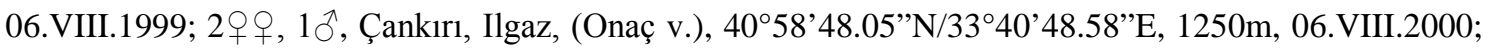
1오, Çankırı, Ilgaz, (Yalaycık v.), 4059’19.30”N/3342’18.16”E, 1100m, 06.VIII.2000; 1, Kastamonu, Centrum, (Ilgaz Mountain National Park), 4103’17.63”N/3343’07.27’E, 2000m, 13.VIII.2000.

Distribution in the world: Afghanistan, Albania, Austria, Belgium, Bulgaria, China, Czech Republic, Denmark, England, Finland, Former Yugoslavia, France, Germany, Greece, Hungary, India, Iran, Italy, Japan, Kazakhstan, Morocco, Netherlands, Norway, Poland, Portugal, Romania, Russia, Siberia, Spain, Sweden, Switzerland, Syria, Turkestan, Turkey [24 and 30].

\section{Family: Pentatomidae Leach, 1815}

\section{Graphosoma lineatum (Linnaeus, 1758)}

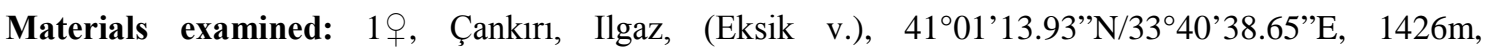
18.VII.1999; 1q, 1§̂, Kastamonu, Centrum, (Çatören v.), 4108’33.89”N/3346’55.82”E, 1200m, 06.VIII.1999; 19, Kastamonu, Centrum, (Çatören v.), 4108’33.89’N/3346’55.82”E, 1200m, 06.VIII.1999; 2우, Kastamonu, Centrum, (Bostan v.), 4106’24.81”N/3344’55.42”E, 1450m, 06.VIII.1999; 1운, Kastamonu, Tosya, (Yukarı İsmailli v.), 4108’47.32”N/3404’10.01”E, 1400m, 07.VIII.1999; 1§̂, Kastamonu, Tosya, (Güney v.), 41¹0’05.60’N/3403’54.17’E, 1250m, 07.VIII.1999.

Distribution in the world: Bulgaria, Iran, North Africa, South Europe, Turkey [20 and 31]. 


\section{Sciocoris (s. str.) sulcatus Fieber 1851}

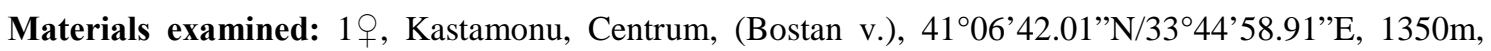
23.V.1999; 1언, Kastamonu, Centrum, (Çatören v.), 4108’33.89”N/3346’55.82”E, 1200m, 18.VII.1999; 2 우우, Kastamonu, Centrum, (Tekke v.), 41¹2’57.97’N/3402’42.40”E, 1400m, 13.VI.2000.

Distribution in the world: Albania, Algeria, Caucasus, Corsica, Cyprus, Former Yugoslavia, Greece, Hungary, Italy, Lebanon, Morocco, Portugal, Romania, Sardinia, Sicily, South Russia, Southern France, Spain, Syria, Transcaucasia, Tunisia, Turkestan, Turkey [20, 24, 25, 30, 31].

\section{Aelia acuminata (Linnaeus, 1758)}

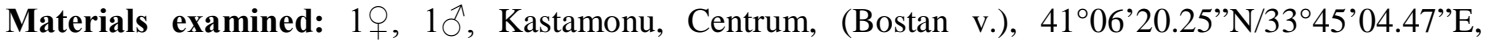
1400m, 06.VI.1999; 2 우, Kastamonu, Centrum, (Beşdeğirmenler v.), 41¹2’05.20”N/3347’52.27”E,

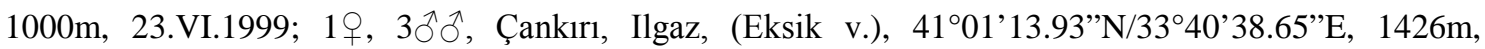
18.VII.1999; 1, Kastamonu, Centrum, (Bostan v.), 4106’24.81”N/3344’55.42”E, 1450m,

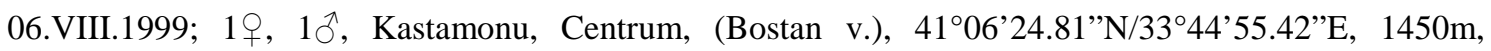

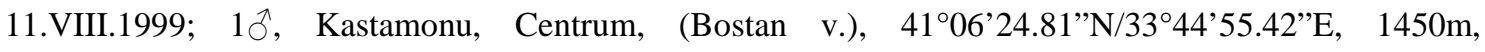

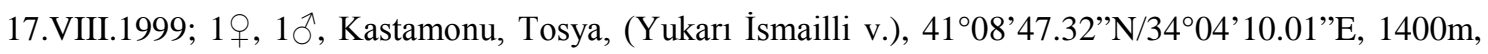

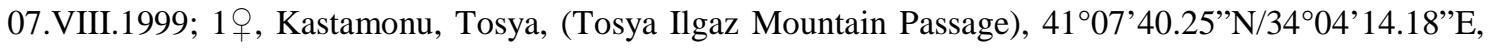
1650m, 07.VIII.1999; 19, Kastamonu, Centrum, (East of Ilgaz Mountain Pass), 4103’54.67”N/3345’08.79”E, 1875m, 11.VI.2000; 1ठ, Çankırı, Ilgaz, (Kırkpınar Plateau), 4100’19.36”N/33³7'14.09”'E, 1800m, 17.IX.2000.

Distribution in the world: Albania, Algeria, Austria, Belarus, Belgium, Bosnia and Herzegovina, Bulgaria, Croatia, Cyprus, Czech Republic, Denmark, England, Estonia, Finland, Former Yugoslavia, France, Germany, Greece, Hungary, Iran, Iraq, Italy, Latvia, Liechtenstein, Lithuania, Luxembourg, Macedonia, Madeira, Malta, Moldova, Morocco, Netherlands, Poland, Portugal, Romania, Russia, Siberia, Sweden, Switzerland, Syria, Tunisia, Turkestan, Turkey, Ukraine [24-26, 33].

\section{Neottiglossa leporina (Herrich-Schäffer, 1830)}

Materials examined: 1 + , Kastamonu, Tosya, (Yukarı İsmailli v.), 4108’47.32”N/3404’10.01”E, 1400m, 07.VIII.1999; 2ᄋํ, 1ठ̋, Çankırı, Ilgaz, (Musaköy v.), 4058’04.05”N/3340’48.35”E, 1100m, 06.VIII.2000; 1ㅇ, Çankırı, Ilgaz, (Yalaycık v.), 4059’19.30”N/3342’18.16”E, 1100m, 06.VIII.2000;

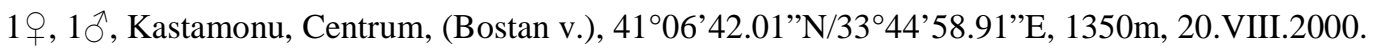

Distribution in the world: Austria, Bulgaria, Caucasus, Corsica, Czech Republic, Former Yugoslavia, Greece, Hungary, Iran, Italy, Kyrgyzstan, Portugal, Romania, Sardinia, Siberia, Sicily, South Russia, Southern France, Spain, Switzerland, Tajikistan, Transcaucasia, Turkestan, Turkey [20 and 25].

\section{Eysarcoris venustissimus (Schrank, 1776)}

Materials examined: 1 + , Kastamonu, Centrum, (Çatören v.), 4108’33.89”N/3346’55.82”E, 1200m, 30.VIII.1999; 19, Kastamonu, Centrum, (Çatören v.), 4108’33.89’N/3346’55.82”E, 1200m, 18.IX.1999; 19, Kastamonu, Centrum, (Ilgaz Mountain National Park Derbent), 4103’50.94’N/3344’38.36”'E, 1800m, 11.VI.2000.

Distribution in the world: Albania, Austria, Baltic States, Belgium, Bulgaria, Czech Republic, Denmark, England, Former Yugoslavia, France, Germany, Hungary, Italy, Japan, Netherlands, Poland, 
Portugal, Romania, Sardinia, Siberia, Sicily, Southern Russia, Spain, Sweden, Switzerland, Turkey, Western Russia [20 and 24].

79. Stagonomus (s. str.) amoenus (Brullé, 1832)

Materials examined: 2 우, Kastamonu, Tosya, (Dipsiz Lake), 4100’55.64’N/3352’34.65’E, 1478m, 12.VII.2000.

Distribution in the world: Afghanistan, Albania, Algeria, Bulgaria, Caucasus, Crete, Cyprus, Czech Republic, Former Yugoslavia, Greece, Hungary, Iran, Iraq, Israel, Italy, Morocco, Portugal, Romania, Sicily, South Russia, Southern France, Spain, Syria, Transcaucasia, Turkey, Turkistan [15, 20, 24, 25, 29, 31].

80. Staria lunata (Hahn, 1835)

Materials examined: 1 , Kastamonu, Centrum, (West of Ilgaz Mountain Pass), 4103’57.67’N/3344’42.31”E, 1875m, 10.VI.2000.

Distribution in the world: Albania, Algeria, Austria, Bulgaria, Caucasus, Corsica, Cyprus, Czech Republic, Former Yugoslavia, Germany, Greece, Hungary, Iran, Israel, Italy, Morocco, Portugal, Romania, Sicily, South Russia, Southern France, Spain, Switzerland, Transcaucasus, Turkey [15, 20, 24, $25,29,31]$.

\section{Peribalus (s. str.) strictus (Fabricius 1803)}

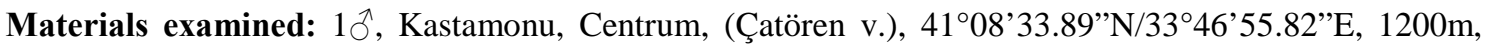
27.V.2000; 1ठ̂, Çankırı, Ilgaz, (Eksik v.), 4101’13.93”N/3340’38.65”E, 1426m, 11.VI.2000.

Distribution in the world: Albania, Algeria, Austria, Baltic countries, Belgium, Bulgaria, Canary Islands, Caucasus, Corsica, Crete, Cyprus, Czech Republic, Denmark, Eastern Russia, England, Finland, Former Yugoslavia, France, Greece, Hungary, Iran, Iraq, Israel, Italy, Netherlands, Norway, Pakistan, Poland, Portugal, Sardinia, Siberia, Sicily, South Russia, Spain, Sweden, Switzerland, Syria, Transcaucasia, Tunisia, Turkestan, Turkey, Western Russia [15, 20, 24, 25, 28, 31].

\section{Palomena prasina (Linnaeus, 1761)}

Materials examined: 1 ㅇ, Kastamonu, Tosya, (Yukarı İsmailli v.), 4108’47.32”N/3404’10.01”E, 1400m, 07.VIII.1999; 2우, 1ठ, Kastamonu, Tosya, (Dipsiz Lake), 4100’55.64”N/3352’34.65”E, 1478m, 12.VII.2000; 1ठ, Kastamonu, Centrum, (Ilgaz Mountain National Park),

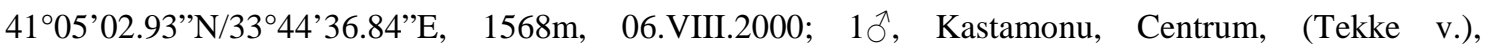
41¹2’57.97’'N/3402’42.40”'E, 1400m, 13.VI.2000.

Distribution in the world: Algeria, Austria, Baltic countries, Belgium, Bulgaria, Caucasus, Czech Republic, Denmark, England, Finland, Former Yugoslavia, France, Germany, Hungary, Ireland, Italy, Morocco, Netherlands, Norway, Poland, Portugal, Romania, Sardinia, Siberia, South Russia, Spain, Sweden, Switzerland, Syria, Transcaucasia, Turkestan, Turkey, Western Russia [20, 24-26, 29, 30].

\section{Carpocoris (s. str.) fuscispinus (Boheman, [1851])}

Materials examined: $1 \hat{\delta}$, Kastamonu, Tosya, (Tosya Ilgaz Mountain Passage), 4107’40.25”N/3404’14.18”E, 1650m, 07.VIII.1999; 1ㅇ, Kastamonu, Centrum, (Ilgaz Mountain Ski Center), 4104’40.67’N/3343’45.54’E, 1800m, 05.VIII.2000; 1ㅇ, Kastamonu, Centrum, (Ilgaz

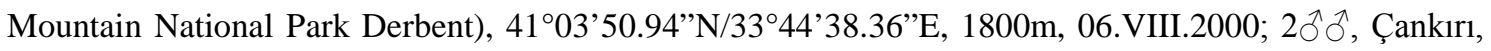




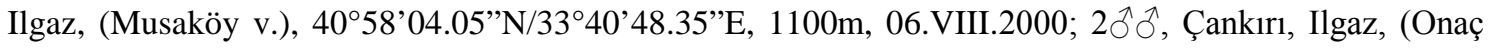
v.), 4058’48.05”N/3340’48.58”E, 1250m, 06.VIII.2000.

Distribution in the world: Afghanistan, Albania, Austria, Bulgaria, Caucasus, China, Corsica, Crete, Cyprus, Czech Republic, Denmark, Egypt, Finland, France, Germany, Greece, Hungary, Iran, Iraq, Israel, Italy, Japan, Kyrgyzstan, Mongolia, Netherlands, Poland, Portugal, Romania, South Russia, Spain, Sweden, Switzerland, Syria, Tajikistan, Transcaucasia, Turkey, Western Russia [15, 16, 20, 24, 25, 29, 33].

\section{Carpocoris (s. str.) pudicus (Poda, 1761)}

Materials examined: 1+, Çankırı, Ilgaz, (Musaköy v.), 4058’04.05”N/3340’48.35”E, 1100m,

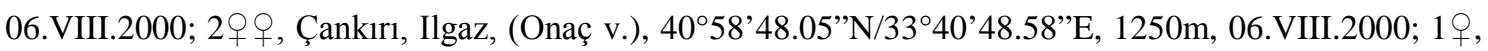
Çankırı, Ilgaz, (Yalaycık v.), 4059’19.30’N/3342’18.16”E, 1100m, 06.VIII.2000.

Distribution in the world: Afghanistan, Albania, Austria, Bulgaria, Caucasus, Corsica, Crete, Cyprus, Czech Republic, Egypt, Former Yugoslavia, Germany, Greece, Hungary, Iran, Iraq, Israel, Italy, Poland, Southern France, Switzerland, Syria, Transcaucasia, Turkey, Turkistan [15, 20, 25, 27, 29, 30].

\section{Carpocoris (s. str.) purpureipennis (De Geer, 1773)}

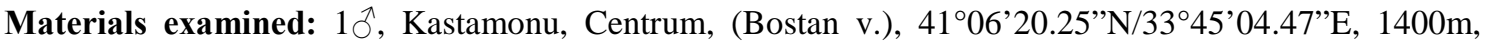
06.VI.1999; 1ðð, Kastamonu, Centrum, (Bostan v.), 4106’20.25”N/3345’04.47”E, 1400m, 23.VI.1999;

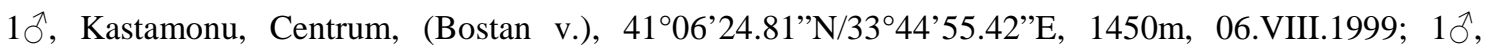
Kastamonu, Centrum, (Ilgaz Mountain National Park), 4105’02.93”N/3344’36.84”E, 1568m,

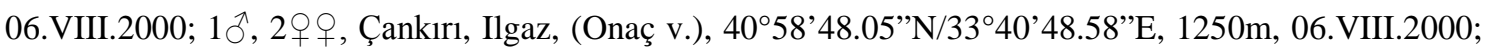

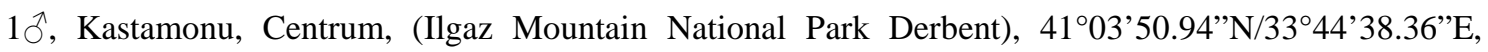
1800m, 06.VIII.2000; 19, Kastamonu, Centrum, (Ilgaz Mountain National Park),

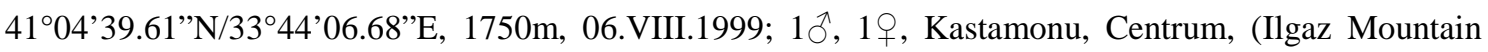

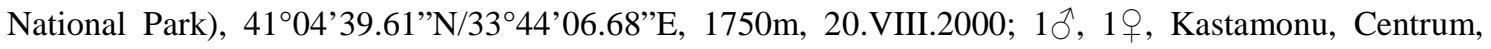

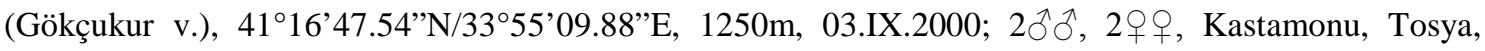

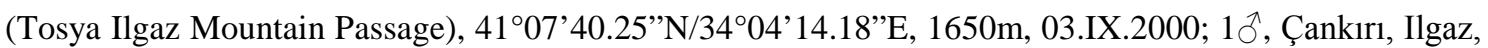

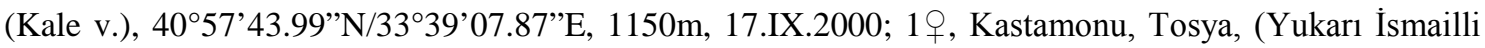

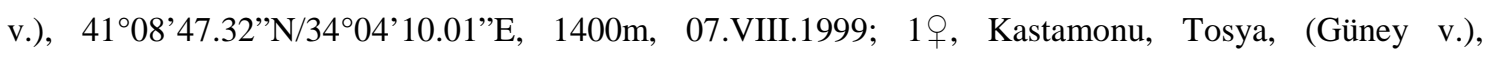
4110’05.60”N/3403’54.17”E, 1250m, 07.VIII.1999; 3우, Kastamonu, Centrum, (Ilgaz Mountain Ski Center), 4104’40.67’N/3343’45.54’E, 1800m, 11.VIII.1999; 1ㅇ, Kastamonu, Centrum, (Çatören v.),

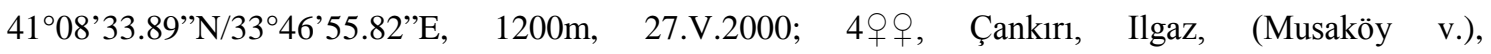
4058’04.05”N/3340’48.35”E, 1100m, 06.VIII.2000; 1q, Çankırı, Ilgaz, (Yalayc1k v.), 4059’19.30”N/3342’18.16”E, 1100m, 06.VIII.2000.

Distribution in the world: Austria, Baltic countries, Belgium, Bulgaria, Corsica, Czech Republic, Denmark, England, Finland, Former Yugoslavia, France, Germany, Hungary, Italy, Manchuria, Mongolia, Netherlands, Norway, Poland, Romania, Siberia, South Russia, Spain, Sweden, Switzerland, Turkey, Western Russia [15 and 20].

\section{Codophila varia (Fabricius, 1787)}

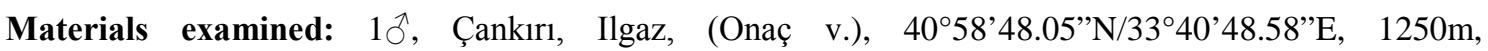
06.VIII.2000. 
Distribution in the world: Albania, Bosnia and Herzegovina, Bulgaria, Canary Islands, Caucasus, Crete, Croatia, Cyprus, Former Yugoslavia, France, Greece, Hungary, Iran, Iraq, Israel, Italy, Kyrgyzstan, Macedonia, Malta, Moldova, North Africa, Northwest Russia, Poland, Portugal, Romania, Spain, Switzerland, Syria, Tajikistan, Transcaucasia, Turkestan, Turkey, Turkmenistan, Ukraine [15, 20, 25, 27, 29- 31].

\section{Dolycoris baccarum (Linnaeus, 1758)}

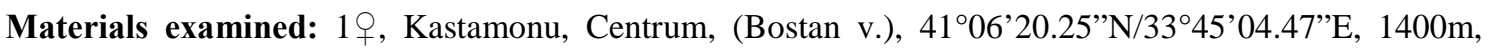

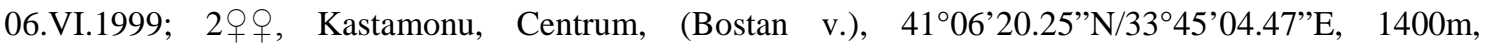

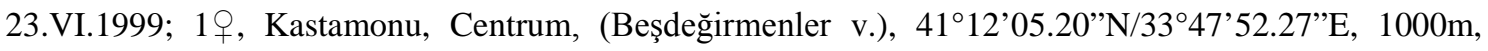

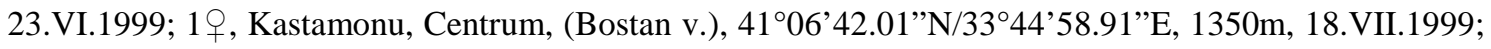

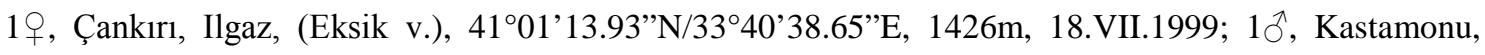

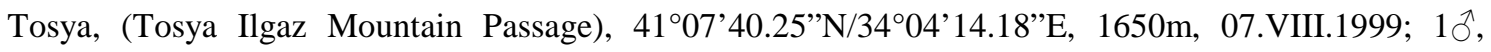

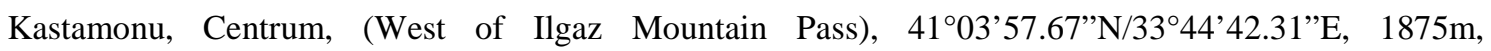

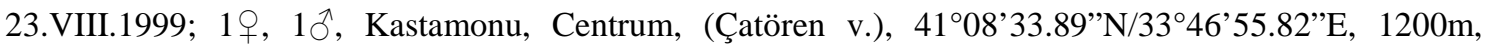

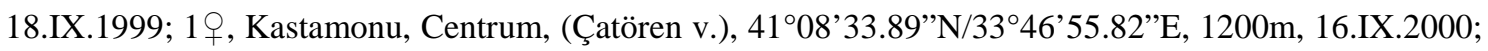

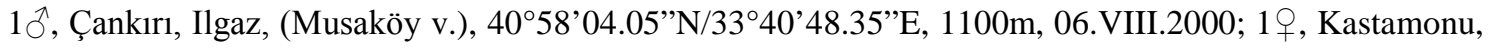
Centrum, (Küçük Hacet Hill), 4104’51.82”N/3348’43.93”E, 2500m, 09.IX.2000; 1우, Çankırı, Ilgaz, (Kırkpınar Plateau), 4100’19.36”N/33॰37’14.09”E, 1800m, 17.IX.2000.

Distribution in the world: Albania, Austria, Belarus, Belgium, Bosnia and Herzegovina, Bulgaria, Caucasus, Crete, Croatia, Cyprus, Czech Republic, Denmark, England, Estonia, Finland, Former Yugoslavia, France, Germany, Greece, Hungary, Iraq, Ireland, Israel, Italy, Japan, Latvia, Liechtenstein, Lithuania, Luxembourg, Macedonia, Madeira, Malta, Moldova, Netherlands, Norway, Poland, Portugal, Romania, Russia, Slovakia, Slovenia, Spain, Sweden, Switzerland, Syria, The Middle East Asia, Transcaucasia, Turkey, Ukraine [15, 20, 25-27, 29-31, 33].

\section{Eurydema (s. str.) oleracea (Linnaeus, 1758)}

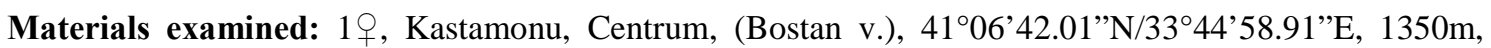

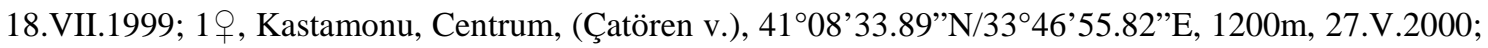

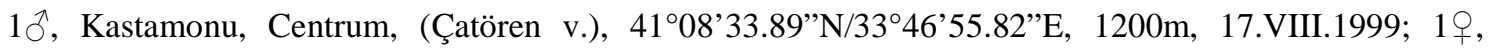

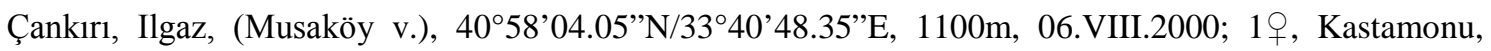

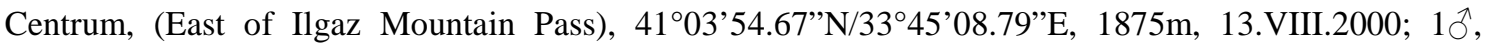

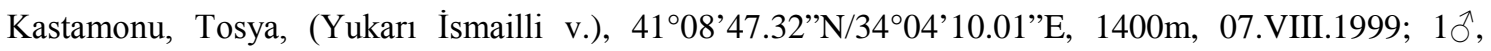

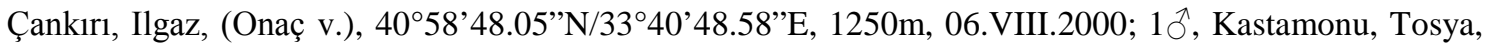

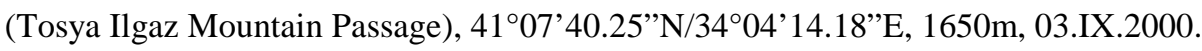

Distribution in the world: Albania, Algeria, Austria, Baltic countries, Belgium, Bulgaria, Caucasus, Corsica, Cyprus, Czech Republic, Denmark, England, Finland, Former Yugoslavia, France, Greece, Hungary, Italy, Kyrgyzstan, Morocco, Netherlands, Norway, Poland, Portugal, Romania, Sardinia, Siberia, Sicily, South Russia, Spain, Sweden, Switzerland, Tajikistan, Transcaucasia, Turkestan, Turkey, Western Russia [20, 25, 26, 29-31, 33].

\section{Eurydema (s. str.) ornata (Linnaeus, 1758)}




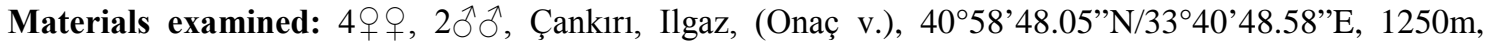
06.VIII.2000.

Distribution in the world: Caucasus, Cyprus, Egypt, Iran, Iraq, Israel, Middle Asia countries, Syria, Transcaucasia, Turkestan, Turkey [15, 20, 25, 27, 30, 31].

\section{Eurydema (Rubrodorsalium) ventralis Kolenati, 1846}

Materials examined: $3 ㅇ++, 3 \widehat{\jmath}$, Çankırı, Ilgaz, (Onaç v.), 4058’48.05”N/3340’48.58”E, 1250m, 06.VIII.2000.

Distribution in the world: Albania, Algeria, Austria, Bulgaria, Caucasus, Corsica, Cyprus, Czech Republic, Egypt, Former Yugoslavia, Greece, Hungary, India, Italy, Malta, Morocco, Poland, Portugal, Romania, Sardinia, Sicily, South Russia, Southern France, Spain, Switzerland, Syria, Transcaucasia, Tunisia, Turkestan, Turkey [20, 24, 25, 29-31].

\section{Nezara viridula (Linnaeus, 1758)}

Materials examined: 1 + , Kastamonu, Centrum, (Çatören v.), 4108’33.89”N/3346’55.82”E, 1200m, 27.V.2000; 1, Kastamonu, Tosya, (Dipsiz Lake), 4100’55.64”N/3352’34.65”E, 1478m, 12.VII.2000.

Distribution in the world: Albania, Algeria, Austria, Azores, Balearic Islands, Bulgaria, Canary Islands, Caucasus, China, Crete, Cyprus, Egypt, Former Yugoslavia, Greece, Hungary, Iran, Iraq, Israel, Italy, Japan, Maeira Islands, Morocco, Portugal, Sardinia, Sicily, Southern France, Spain, Switzerland, Syria, Transcaucasia, Tunisia, Turkey [20 and 25].

\section{Piezodorus lituratus (Fabricius, 1794)}

Materials examined: 1 q, Çankırı, Ilgaz, (Eksik v.), 4101'13.93”N/3340’38.65”E, 1426m, 11.VI.2000; 1q, Çankırı, Ilgaz, (Musaköy v.), 4058’04.05”N/3340’48.35”E, 1100m, 06.VIII.2000; 1ठ̂, Kastamonu, Tosya, (Dipsiz Lake), 4100’55.64”N/3352’34.65”E, 1478m, 12.VII.2000; 10̂, Çankırı, Ilgaz, (Onaç v.),

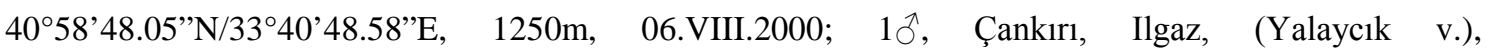
4059’19.30”N/3342’18.16”E, 1100m, 06.VIII.2000; 1ㅇ, Kastamonu, Tosya, (Ekincik v.), 4104’01.26”N/3400’52.24”E, 1000m, 03.IX.2000.

Distribution in the world: Albania, Algeria, Austria, Balearic Islands, Belgium, Bulgaria, Corsica, Crete, Cyprus, Czech Republic, England, Former Yugoslavia, France, Germany, Greece, Hungary, Iran, Ireland, Italy, Morocco, Netherlands, Poland, Portugal, Romania, Sardinia, Scotland, Sicily, South Russia, Spain, Sweden, Switzerland, Syria, Tunisia, Turkestan, Turkey, Western Russia [15, 20, 25, 26, 30].

\section{Rhaphigaster nebulosa (Poda, 1761)}

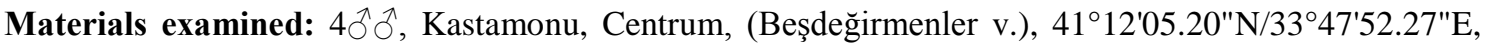
1000m, 23.VI.1999.

Distribution in the world: Afghanistan, Albania, Algeria, Austria, Balearic Islands, Belgium, Bulgaria, Caucasus, Crete, Cyprus, Czech Republic, Former Yugoslavia, France, Germany, Greece, Hungary, Iran, Iraq, Israel, Italy, Mongolia, Morocco, Netherlands, Poland, Portugal, Romania, Sardinia, Sicily, Spain, Switzerland, Syria, Turkestan, Turkey, Western Russia [20, 25, 27]. 


\section{Discussion}

In this study, a total of 474 Heteroptera were collected between May 1999 and Semtember 2000, in order to determine the Heteroptera fauna of the Ilgaz Mountains. As result of diagnostic species collected have been identified 93 species belonging to the genus 73 of the 16 families (Table 1), which is \% 6.09 percentage of the number of Heteropteroids recorded in Turkey. 75 of 93 taxa are new for the Heteroptera fauna of Ilgaz Mountains.

It has been reported four species and subspecies in the genus Velia in Turkey so far. Distribution in the Turkey of this species and subspecies are as follows: Velia (Plesiovelia) affinis affinis Kolenati, 1857: Adana, Amasya, Ankara, Antalya, Artvin, Bursa, Giresun, Hatay, Isparta, Konya, Malatya, Mersin, Niğde, Sivas [3, 35, 36, 37]. Velia (Plesiovelia) affinis filippii Tamanini, 1947: Adana, Akdeniz Bölgesi, Antalya, Aydın, Burdur, Denizli, Isparta, Kırklareli, Muğla, Sinop [3, 36, 38]. Velia (Plesiovelia) kiritshenkoi Tamanini, 1958: Giresun, Kahramanmaraş, Sivas, Tokat, Van, Yozgat [35, 36, 37, 39]. Velia (Plesiovelia) mancinii lyciae Tamanini, 1955: Denizli [36]. Velia (Plesiovelia) mariae Tamanini, 1971: Denizli [36]. Velia (Plesiovelia) rhadamantha rhadamantha Hoberlandt, 1941: Kırklareli [36].Velia nervosa Horváth, 1896: Akdeniz Bölgesi [3]. Velia (Plesiovelia) caprai Tamanini 1947: Antalya, Aydın, Burdur, Isparta, Kırşehir, Muğla [38, 39]. This species is widespread in all of Europe and has expanded its distribution until the very end of the West Palearktik Ilgaz Mountains in Turkey. This species that common in Europe is likely to be available in Ilgaz Mountains, because of in the Euro Siberian zoogeographic region.

Hoberlandt [6] listed 6 species belonging to 4 families (Notonectidae, Gerridae, Corixidae, Saldidae), Lodos et al. [8], Wagner [10], Yardım [11] listed 37 species belonging to Miridae, Lodos and Önder [7] listed 6 species belonging to Tingidae, Boz [4] listed 1 species belonging to Reduviidae, Lodos et al. [9] listed 14 species belonging to Lygaeidae, Fent [5] listed 18 species belonging to 3 family (Pentatomidae, Scutelleridae, Cydnidae) from Ilgaz Mountains. Consequently, occurrence of 64 species and subspecies previously reported from this area has not been confirmed during the fieldwork of this study. These 64 species and subspecies have small samples, the presence of this species in different habitats has made it difficult for us to find. Thus, these species and subspecies has not been reached our studies. In addition to 18 known species belonging to 6 families have been found in this study [4-6, 8-11].

With earlier publications, the total number of Heteroptera taxa recorded from this area is 157 species representing 20 families. The number of species for each province is given in Table 1 .

Figure 2 displays the Heteropteran assemblages of whole region and its relative frequency per taxa. The 93 species and subspecies, found represented sixten families. Family Miridae induced the majority of heteropteroids with 51 taxa (\% 32,48), while Lygaeidae 24 taxa (\% 15,29), Pentatomidae 24 taxa (\%15,29), Rhopalidae 11 taxa $(\% 7,01)$, Scutelleridae 8 taxa $(\% 5,10)$. 


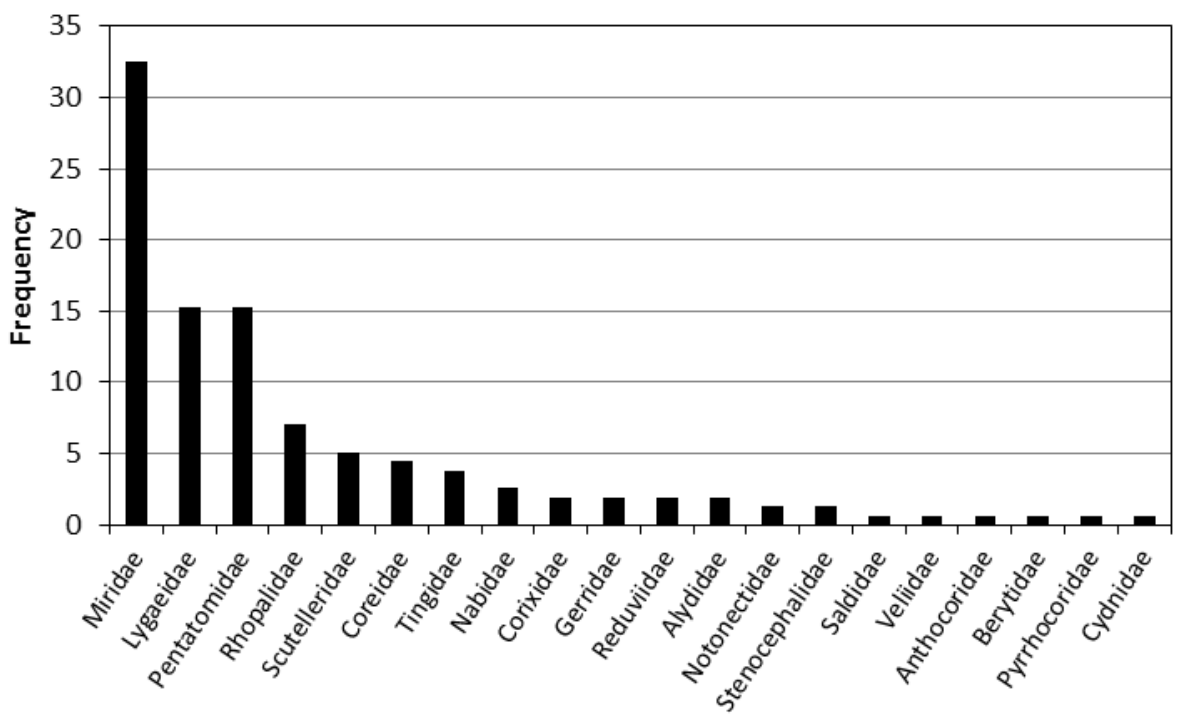

Figure 2. The frequently per species of sixteen families in the Ilgaz Mountains.

These results suggest that our country has quite a different geographic and climatic characteristics reveals the fact that more species found in each local region of Turkey in detail illustrates the need for local operation.

\section{References}

[1] Schuh R. T., Slater J. A., "True bugs of the World (Hemiptera: Heteroptera)” Cornell University Press, 336 pp, Ithaca, 1995.

[2] Henry T. J., "Biodiversity of Heteroptera, 223-263. In: Foottit RG, Adler PH (Eds) Insect Biodiversity”, Science and Society Chichester, Wiley-Blackwell, UK, 632pp, 2009.

[3] Önder F., Karsavuran K., Tezcan S., Fent M., “Türkiye Heteroptera (Insecta) Kataloğu” Meta Basım Matbaacılık Hizmetleri, 164s, İzmir, 2006.

[4] Boz E., "Türkiye Harpactorinae (Heteroptera: Reduviidae) faunası üzerinde sistematik çalışmalar" Ege Üniversitesi Fen Bilimleri Enstitüsü Bitki Koruma Anabilim Dalı, Yüksek Lisans tezi, 116s, İzmir, 1992.

[5] Fent M., "Contributions to Pentatomoidea (Heteroptera) Fauna of Western Black Sea Region with a New Record for Anatolian Fauna: Neottiglossa lineolata (Mulsant \& Rey, 1852)” Journal of The Entomological Research Society, 12(1), 53-65, 2010.

[6] Hoberlandt L., "Results of the Zoological Scientific Expedition of the National Museum in Praha to Turkey. Hemiptera-Heteroptera I. The Aquatic and Semiaquatic Heteroptera of Turkey” Acta Entomologica Musei Nationalis Pragae, 26, 1-71, 1948.

[7] Lodos N., Önder F., "Contribution to the Turkish Pentatomidae (Heteroptera) IV. Asopinae (Amyont \& Searville, 1843)”, Türkiye Bitki Koruma Dergisi, 7(4), 221-230, 1983.

[8] Lodos N., Önder F., Pehlivan E., Atalay R., Erkin E., Karsavuran Y., Tezcan S., Aksoy S., "Faunistic studies on Miridae of Western Black Sea, Central Anatolia and Mediterranean Regions of Turkey” Meta Basım, 85pp, İzmir, 2003. 
[9] Lodos N., Önder F., Pehlivan E., Atalay R., Erkin E., Karsavuran Y., Tezcan S., Aksoy S., "Faunistic studies on Lygaeidae (Heteroptera) of Western Black Sea, Central Anatolia and Mediterranean Regions of Turkey” Ege Üniversitesi Basımevi, 58pp, İzmir, 1999.

[10] Wagner E., "Die Miridae Hahn, 1831, des Mittelmeerraumes und der Makaronesischen Inseln (Hemiptera, Heteroptera)", Teil 1, Entomologische Abhandlungen Museum Tierkunde Dresden, (Suppl.) 37, 1-484, 1970-1971.

[11] Yardım E. N., "Türkiye Stenodemini (Heteroptera: Miridae: Mirinae) Faunası Üzerine Sistematik Araştırmalar" Ege Üniversitesi, Fen Bilimileri Enstisüsü, Yüksek Lisans tezi, 113s, İzmir, 1990.

[12] Küçükbasmacı İ., "Ilgaz Dağları Heteroptera Türlerinin Faunistik Yönden Araştırılması”, Gazi Üniversitesi, Fen Bilimleri Enstitüsü Biyoloji Anabilim Dalı, Yüksek Lisans tezi, 168s, Ankara, 2001.

[13] Ahmad I., Abbas N., "Pyrrhocoris group (Hemiptera, Heteroptera, Pyrrhocorinae) with description of a new genus and species from Indo-Pakistan subcontinent and relationships" Türkiye Bitki Koruma Dergisi, 10(2), 67-87, 1986.

[14] Kerzhner I. M., Yachevskii T. L., “Order Hemiptera (Heteroptera), 851-1118. In: Keys to the Insects of the European USSR I. Apterygota, Palaeoptera, Hemimetabola” Edited by Bei-Bienko, G. Ya., Israel Program for Scientific Translations, Jerusalem, 1214pp, 1967.

[15] Kıyak S., "Systematisch-Ökologische Untersuchungen Über Die Wanzen (Insecta: Heteroptera) Aus Dem Gebiet Hazar-See, Maden und Ergani (Prov. Elazığ)" Gazi Üniversitesi Fen Edebiyat Fakültesi Biyoloji Dergisi, 1, 43-95, 1990.

[16] Lodos N., Önder F., “Türkiye Pentatomidae (Heteroptera) üst familyası üzerine araştırmalar", Türkiye Bitki Koruma Dergisi, 2(4), 195-239, 1978.

[17] Pehlivan E., "Türkiye Stenocephalidae, Rhopalidae ve Alydidae (Heteroptera: Coreoidea) Faunası Üzerinde Sistematik Araştırmalar" Ege Üniversitesi Ziraat Fakültesi Yayınları, No: 410, 189s, İzmir, 1981.

[18] Stichel W., "Illustrierte Bestimmungstabellen der Wanzen II. Europa (Hemiptera - Heteroptera Europae)”, I, Hermsdorf: Selbstverlag, 1-168, Berlin, 1955.

[19] Stichel W., "Illustrierte Bestimmungstabellen der Wanzen II. Europa (Hemiptera - Heteroptera Europae)”, II, Hermsdorf: Selbstverlag, 170-907, Berlin, 1956-1958.

[20] Stichel W., "Illustrierte Bestimmungstabellen der Wanzen II. Europa (Hemiptera - Heteroptera Europae)”, IV, Hermsdorf: Selbstverlag, 1-830, Berlin, 1957-1962.

[21] Stichel W., "Illustrierte Bestimmungstabellen der Wanzen II. Europa (Hemiptera - Heteroptera Europae)”, III, Hermsdorf: Selbstverlag, 1-428, Berlin, 1958-1960.

[22] Wagner E., "Die Miridae Hahn, 1831, des Mittelmeerraumes und der Makaronesischen Inseln (Hemiptera, Heteroptera)", Teil 3, Entomologische Abhandlungen Museum Tierkunde Dresden, (Suppl.) 40, 1-483, 1973.

[23] Aukema B., Rieger C., "Catalogue of the Heteroptera of the Palaearctic Region, Vol. I" The Nederlands Entomological Society, 222pp, Amsterdam, 1995.

[24] Lodos N., Önder F., Pehlivan E., Erkin E., Karsavuran Y., Aksoy S., "Orta Anadolu ve Bat1 Karadeniz Bölgelerinin Böcek Faunasının Tespiti Üzerine Çalışmalar, Curculionidae, 
Scarabeidae (Coleoptera); Pentatomidae, Scutelleridae, Lygaeidae, Miridae (Heteroptera)”, TÜBİTAK, TOAG-336 Nolu proje Kesin Raporu, 51s, Doğa Bilim Dergisi, Ser. D2, 7 (3), 207212, 1983.

[25] Hoberlandt L., "Results of the Zoological Scientific Expedition of the National Museum in Praha to Turkey-18: Hemiptera IV: Terrestriale Hemiptera-Heteroptera of Turkey” Acta Entomologica Musei Nationalis Pragae, 3, 274, 1955.

[26] Kıyak S., Çağlar S. S., "Über Heteropteren Arten von Nord-und Nordostanatolien" Miscellaneous Papers, 11, 2-8, 1991.

[27] Önder F., “Türkiye'nin Mymemorphic Heteroptera Türleri Üzerine Araştırmalar” Ege Üniversitesi Ziraat Fakültesi Bitki Koruma Bölümü, Turkey II. Entomoloji Kongresi Ocak, 363371, Adana, 1992.

[28] Önder F., Ünal E., Ünal A., "Heteropterous insects collected by lighttraps in Edirne (Turkey)" Türkiye Bitki Koruma Dergisi, 8(4), 215-225, 1984.

[29] Tuatay N., Kalkandelen A., (Çağatay) Aysev, N., "Nebat Koruma Müzesi Böcek Kataloğu (1961-1971)” T.C. Tarım Bakanlı̆̆ Zirai Mücadele ve Zirai Karantina Genel Müdürlüğü Yayınları Meslekî Kitaplar Serisi, 2-12, Ankara, 1972.

[30] Kiyak S., “Über terrestrische Wanzenarten Von Soğuksu National Park”, Priamus, 6(3/4), 131156, 1993.

[31] Lodos N., Önder F., Pehlivan E., Atalay R., "Ege ve Marmara Bölgesi Zararlı Böcek Faunasının Tespiti Üzerine Çalışmalar, Curculionidae, Scarabeidae (Coleoptera); Pentatomidae, Lygaeidae, Miridae (Heteroptera)” T.C. Gıda-Tarım ve Hayvancılık Bakanlı̆̆ı Zirai Mücadele ve Karantina Genel Müd. Basimevi, 301s, Ankara, 1978.

[32] Seidenstücker G., “Anadolu'dan Heteropterler II.” İstanbul Üniversitesi Fen Fakültesi Mecmuası, Seri B, 23(1-2), 119-129, 1958.

[33] Altınayar G., "Orta Anadolu Bölgesi tahıl tarlalarındaki böcek faunasının saptanması üzerinde çalışmalar” Bitki Koruma Bülteni, 21(2), 54-88, 1981.

[34] Aysev N., "Ege Bölgesinde Lygaeidae Familyası Üzerinde Sistematik Çalışmalar” Güven Matbaas1, Ankara, 1974.

[35] Dursun A., “Additional records of Gerromorpha (Hemiptera: Heteroptera) and redescription of Rhagovelia nigricans nigricans (Burmeister, 1835) from Anatolia (Turkey)” Turkish Journal of Zoology, 36(5), 652-661, 2012.

[36] Fent, M., Kment, P., Çamur Elipek, B., Kırgız, T., "Annotated catalogue of Enicocephalomorpha, Dipsocoromorpha, Nepomorpha, Gerromorpha and Leptopodomorpha (Hemiptera: Heteroptera) of Turkey with new records” Zootaxa, 2856: 1-84, 2011.

[37] Tamanini, L., “Osservazioni sulla geonemia delle Velia orientali e descrizione di una nuova species. XX Contributo allo studio del genre Velia Latr. (Heteroptera, Veliidae)” Bollettino della Società Entomologica Italiana, 102, 30-35, 1970.

[38] Kıyak, S., Salur, A., Canbulat, S., “Gerromorpha and Leptopodomorpha (Insecta; Heteroptera) Fauna of Southwest Anatolia” Turkish Journal of Zoology, 32, 309-326, 2008.

[39] Kıyak, S., Özsaraç, Ö., "Cheklist of aquatic and semiaquatic Heteroptera of Turkey, with a new record” Journal of the Entomological Research Society, 3, 17-32, 2001. 
Table 1. List of species of Heteroptera recorded from Ilgaz Mountains.

\begin{tabular}{|c|c|c|}
\hline No & Species & Remarks $^{*}$ \\
\hline \multicolumn{3}{|c|}{ Corixidae } \\
\hline 1 & Corixa punctata (Illiger, 1807) & - \\
\hline 2 & Hesperocorixa occulta (Lundblad, 1929) & - \\
\hline 3 & Sigara (Retrocorixa) limitata limitata (Fieber, 1848) & ロ \\
\hline \multicolumn{3}{|c|}{ Notonectidae } \\
\hline 4 & Notonecta (s. str.) glauca glauca Linnaeus, 1758 & - \\
\hline 5 & Notonecta marmorea Fabricius, 1803 & $\mathbf{\Delta}$ \\
\hline \multicolumn{3}{|c|}{ Gerridae } \\
\hline 6 & Gerris (s. str.) costae costae (Herrich-Schäffer, 1850) & $\boldsymbol{\Delta \Delta}$ \\
\hline 7 & Gerris (Gerriselloides) lateralis Schummel, 1832 & $\Delta$ \\
\hline 8 & Aquarius najas (De Geer, 1773) & $\Delta$ \\
\hline \multicolumn{3}{|c|}{ Saldidae } \\
\hline 9 & Saldula saltatoria (Linnaeus, 1758) & - \\
\hline \multicolumn{3}{|c|}{ Veliidae } \\
\hline 10 & Velia (Plesiovelia) saulii Tamanini, 1947 & $\Delta$ \\
\hline \multicolumn{3}{|c|}{ Miridae } \\
\hline 11 & Fulvius punctumalbum (Rossi, 1790) & $\mathbf{\Delta}$ \\
\hline 12 & Bothynotus pilosus (Boheman, 1852) & $\Delta$ \\
\hline 13 & Deraeocoris (s. str.) rutilus (Herrich-Schäffer, [1838]) & $\mathbf{\Delta} \mathbf{\Delta}$ \\
\hline 14 & Monalocoris (s. str.) filicis (Linnaeus, 1758) & $\Delta$ \\
\hline 15 & Megalocoleus tanaceti (Fallén, 1807) & $\Delta$ \\
\hline 16 & Plagiotylus dispar Reuter, 1899 & $\Delta$ \\
\hline 17 & Stenodema (s. str.) laevigatum (Linnaeus, 1758) & $\mathbf{\Delta \Delta}$ \\
\hline 18 & Notostira erratica (Linnaeus, 1758) & $\Delta$ \\
\hline 19 & Leptopterna dolabrata (Linnaeus, 1758) & $\mathbf{\Delta}$ \\
\hline 20 & Adelphocoris vandalicus (Rossi, 1790) & $\boldsymbol{\Delta}$ \\
\hline 21 & Closterotomus reuteri (Horvath, 1882) & $\Delta$ \\
\hline 22 & Grypocoris (Turciocoris) heinzi Wagner, 1966 & $\boldsymbol{\Delta \Delta}$ \\
\hline 23 & Aphanosoma italicum Costa, 1842 & $\boldsymbol{\Delta}$ \\
\hline 24 & Brachycoleus decolor Reuter, 1887 & $\Delta$ \\
\hline 25 & Stenotus binotatus (Fabricius, 1794) & $\Delta$ \\
\hline 26 & Liocoris tripustulatus (Fabricius, 1781) & $\Delta$ \\
\hline 27 & Charagochilus (s. str.) gyllenhalii (Fallén, 1807) & $\Delta$ \\
\hline 28 & Apolygus lucorum (Mayer-Dür, 1843) & $\mathbf{\square}$ \\
\hline 29 & Notostira elongata (Geoffroy, 1785) & $\mathbf{\square}$ \\
\hline 30 & Orthops (s. str.) kalmii (Linnaeus, 1758) & $\mathbf{\square}$ \\
\hline & Phytocoris irroratus Linnavuori, 1965 & घ \\
\hline
\end{tabular}




\begin{tabular}{|c|c|c|}
\hline 32 & Phytocoris (s. str.) pini Kirschbaum, 1856 & - \\
\hline 33 & Phytocoris (Exophytocoris) tauricola Linnavuori, 1965 & - \\
\hline 34 & Atractotomus magnicornis (Fallén, 1807) & - \\
\hline 35 & Atractotomus mali (Meyer-Dür, 1843) & - \\
\hline 36 & Chlamydatus (Euattus) pullus (Reuter, 1870) & - \\
\hline 37 & Plagiognathus (s. str.) arbustorum (Fabricius, 1794) & - \\
\hline 38 & Plagiognathus (s. str.) bipunctatus Reuter, 1883 & घ \\
\hline 39 & Lepidargyrus ancorifer (Fieber, 1858) & - \\
\hline 40 & Psallus (Pityopsallus) pinicola Reuter, 1875 & - \\
\hline 41 & Psallus (s. str.) varians (Herrich-Schäffer, 1841) & - \\
\hline 42 & Deraeocoris (Knightocapsus) lutescens (Schilling, 1837) & - \\
\hline 43 & Deraeocoris (s. str.) ruber (Linnaeus, 1758) & - \\
\hline 44 & Deraeocoris (Camptobrochis) serenus (Douglas \& Scott, 1868) & - \\
\hline 45 & Calocoris affinis (Herrich-Schäffer, 1835) & - \\
\hline 46 & Calocoris roseomaculatus angularis (Fieber 1864) & - \\
\hline 47 & Closterotomus fulvomaculatus (De Geer 1773) & - \\
\hline 48 & Exolygus pratensis (Linnaeus, 1758) & - \\
\hline 49 & Lygus rugulipennis Poppius, 1911 & घ \\
\hline 50 & Adelphocoris lineolatus (Goeze, 1778) & - \\
\hline 51 & Dichrooscytus seidenstueckeri Josifov, 1974 & - \\
\hline 52 & Dichrooscytus valesianus Fieber, 1861 & घ \\
\hline 53 & Polymerus (Poeciloscytus) vulneratus (Wolff, 1801) & घ \\
\hline 54 & Dryophilocoris (Camarocyphus) persimilis (Puton, 1895) & - \\
\hline 55 & Orthotylus (Pinocapsus) cupressi Reuter, 1883 & - \\
\hline 56 & Orthotylus (Melanotrichus) flavosparsus (Sahlberg, 1841) & - \\
\hline 57 & Orthotylus (s. str.) quercicola Reuter, 1885 & - \\
\hline 58 & Amblytylus longirostris (Jorden, 1947) & घ \\
\hline 59 & Oncotylus (Cylindromelus) setulosus (Herrich-Schäffer, 1837) & - \\
\hline 60 & Sthenarus collaris Wagner, 1975 & - \\
\hline & Sthenarus roseri (Herrich-Schäffer, 1838) & ロ \\
\hline \multicolumn{3}{|c|}{ Anthocoridae } \\
\hline & Anthocoris nemorum (Linnaeus, 1761) & $\boldsymbol{\Delta}$ \\
\hline \multicolumn{3}{|c|}{ Reduviidae } \\
\hline & Rhynocoris (s. str.) punctiventris (Herrich-Schäffer, 1846) & $\boldsymbol{\Delta}$ \\
\hline 64 & Sphedanolestes (s. str.) pulchellus (Klug, 1830) & $\boldsymbol{\Delta}$ \\
\hline & Nagusta goedeli (Kolenati, 1857) & $\boldsymbol{\Delta \Delta}$ \\
\hline \multicolumn{3}{|c|}{ Nabidae } \\
\hline 66 & Himacerus (s. str.) apterus (Fabricius, 1798) & $\boldsymbol{\Delta}$ \\
\hline 67 & Nabis (Tropiconabis) capsiformis Germar, 1838 & $\boldsymbol{\Delta}$ \\
\hline 68 & Nabis (s. str.) pseudoferus Remane, 1949 & $\boldsymbol{\Delta}$ \\
\hline
\end{tabular}


69 Nabis (Aspilaspis) viridulus Spinola, 1837

Tingidae

70 Acalypta marginata (Wolff, 1804)

71 Dictyonata tricornis tricornis (Schrank, 1901)

72 Lasiacantha capucina (Germar, 1837)

73 Tingis (s. str.) cardui cardui (Linnaeus 1758)

74 Catoplatus dilatatus (Jakovlev, 1880)

75 Dictyla nassata (Puton, 1874)

Berytidae

76 Neides tipularius (Linnaeus, 1758)

\section{Lygaeidae}

77 Lygaeus equestris (Linnaeus, 1758)

78 Spilostethus saxatilis (Scopoli, 1763)

79 Melanocoryphus albomaculatus (Goeze, 1778)

80 Ortholomus punctipennis (Herrich-Schäffer, 1838)

81 Heterogaster affinis Herrich-Schäffer, 1835

82 Macroplax fasciata fasciata (Herrich-Schäffer, 1835)

83 Gastrodes grossipes (De Geer, 1773)

84 Emblethis griseus (Wolff, 1802)

85 Peritrechus geniculatus (Hahn, 1832)

86 Peritrechus gracilicornis Puton, 1887

87 Rhyparochromus pini (Linnaeus, 1758)

88 Beosus maritimus (Scopoli, 1763)

89 Orsillus depressus (Mulsant \& Rey 1852)

90 Beosus quadripunctatus (Müller, 1766)

91 Geocoris (Piocoris) erythrocephalus (Lepeletier \& Serville, 1825)

92 Platyplax inermis (Rambur 1839)

93 Tropidothorax leucopterus (Goeze, 1778)

94 Nysius graminicola (Kolenati, 1845)

95 Metopoplax fuscinervis Stal, 1872

96 Oxycarenus (s. str.) hyalinipennis (Costa, 1843)

97 Oxycarenus longiceps Wagner, 1955

98 Megalonotus praetextatus (Herrich-Schäffer, 1835)

99 Megalonotus sabulicolus (Thomson, 1870)

100 Peritrechus ambiguus Horvath, 1888

Pyrrhocoridae

101 Pyrrhocoris apterus (Linnaeus, 1758)

Stenocephalidae

102 Dicranocephalus agilis (Scopoli, 1763)

103 Dicranocephalus albipes (Fabricius, 1781) 


\begin{tabular}{|c|c|c|}
\hline \multicolumn{3}{|c|}{ Coreidae } \\
\hline & Gonocerus acuteangulatus (Goeze, 1778) & $\boldsymbol{\Delta}$ \\
\hline & Gonocerus juniperi Herrich-Schäffer, 1839 & $\Delta$ \\
\hline & Coreus marginatus marginatus (Linnaeus, 1758) & $\boldsymbol{\Delta}$ \\
\hline & Syromastus rhombeus (Linnaeus, 1767) & $\Delta$ \\
\hline & Enoplops scapha (Fabricius, 1794) & $\Delta$ \\
\hline & Ceraleptus gracilicornis (Herrich-Schäffer, 1835) & $\Delta$ \\
\hline & Coriomeris hirticornis (Fabricius, 1794) & $\Delta$ \\
\hline \multicolumn{3}{|c|}{ Alydidae } \\
\hline & Alydus calcaratus (Linnaeus, 1758) & $\Delta$ \\
\hline & Camptopus lateralis (Germar, 1817) & $\Delta$ \\
\hline 113 & Camptopus tragacanthae (Kolenati, 1845) & $\Delta$ \\
\hline \multicolumn{3}{|c|}{ Rhopalidae } \\
\hline & Corizus hyoscyami hyoscyami (Linnaeus, 1758) & $\boldsymbol{\Delta}$ \\
\hline & Liorhyssus hyalinus (Fabricius, 1794) & $\Delta$ \\
\hline & Rhopalus (s. str.) conspersus (Fieber, 1837) & $\Delta$ \\
\hline & Rhopalus (Aeschyntelus) maculatus (Fieber 1837) & $\Delta$ \\
\hline & Rhopalus (s. str.) parumpunctatus Schilling, 1829 & $\Delta$ \\
\hline & Rhopalus (s. str.) rufus Schilling, 1829 & $\Delta$ \\
\hline & Stictopleurus crassicornis (Linnaeus, 1758) & $\Delta$ \\
\hline & Stictopleurus pictus (Fieber, 1861) & $\Delta$ \\
\hline & Maccevethus caucasicus (Kolenati, 1845) & $\Delta$ \\
\hline 123 & Maccevethus persicus Jakovlev, 1881 & $\Delta$ \\
\hline & Chorosoma schillingii (Schilling, 1829) & $\Delta$ \\
\hline \multicolumn{3}{|c|}{ Scutelleridae } \\
\hline 125 & Odontotarsus caudatus (Burmeister, 1835) & $\Delta$ \\
\hline & Odontotarsus robustus Jakovlev, [1884] & $\Delta$ \\
\hline & Odontotarsus impictus Jakovlev, 1886 & - \\
\hline & Eurygaster hottentotta (Fabricius, 1775) & $\Delta$ \\
\hline & Eurygaster maura (Linnaeus, 1758) & $\because \boldsymbol{\Delta}$ \\
\hline & Eurygaster dilaticollis Dohrn, 1860 & - \\
\hline & Eurygaster schreiberi Montandon, 1885 & $\Delta$ \\
\hline 132 & Eurygaster testudinaria (Geoffroy, 1785) & $\because \boldsymbol{\Delta}$ \\
\hline \multicolumn{3}{|c|}{ Pentatomidae } \\
\hline 133 & Graphosoma lineatum (Linnaeus, 1758) & $\boldsymbol{\square} \boldsymbol{\Delta}$ \\
\hline 134 & Graphosoma semipunctatum (Fabricius, 1775) & - \\
\hline 135 & Sciocoris (s. str.) sulcatus Fieber 1851 & $\Delta$ \\
\hline & Aelia acuminata (Linnaeus, 1758) & $\because \boldsymbol{\Delta}$ \\
\hline 137 & Aelia rostrata Boheman, 1852 & - \\
\hline 138 & Neottiglossa leporina (Herrich-Schäffer, 1830) & $\Delta$ \\
\hline
\end{tabular}




\begin{tabular}{|c|c|c|}
\hline 139 & Eysarcoris venustissimus (Schrank, 1776) & $\mathbf{\Delta}$ \\
\hline 140 & Stagonomus (s. str.) amoenus (Brullé, 1832) & $\Delta$ \\
\hline 141 & Staria lunata (Hahn, 1835) & $\mathbf{\Delta}$ \\
\hline 142 & Peribalus (s. str.) strictus (Fabricius 1803) & $\Delta$ \\
\hline 143 & Palomena prasina (Linnaeus, 1761) & $\boldsymbol{\Delta \Delta}$ \\
\hline 144 & Carpocoris (s. str.) fuscispinus (Boheman, [1851]) & $\mathbf{\Delta}$ \\
\hline 145 & Carpocoris (s. str.) pudicus (Poda, 1761) & $\Delta$ \\
\hline 146 & Carpocoris (s. str.) purpureipennis (De Geer, 1773) & $\boldsymbol{\Delta \Delta}$ \\
\hline 147 & Carpocoris (s. str.) melanocerus (Mulsant \& Rey, 1852) & - \\
\hline 148 & Coptosoma scutellatum (Geoffroy, 1785) & $\mathbf{\square}$ \\
\hline 149 & Codophila varia (Fabricius, 1787) & $\mathbf{\Delta} \mathbf{\Delta}$ \\
\hline 150 & Dolycoris baccarum (Linnaeus, 1758) & $\mathbf{\Delta}$ \\
\hline 151 & Eurydema (s. str.) oleracea (Linnaeus, 1758) & $\mathbf{\Delta} \mathbf{\Delta}$ \\
\hline 152 & Eurydema (s. str.) ornata (Linnaeus, 1758) & $\mathbf{\Delta \Delta}$ \\
\hline 153 & Eurydema (Rubrodorsalium) ventralis Kolenati, 1846 & $\Delta$ \\
\hline 154 & Nezara viridula (Linnaeus, 1758) & $\boldsymbol{\Delta}$ \\
\hline 155 & Piezodorus lituratus (Fabricius, 1794) & 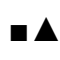 \\
\hline 156 & Rhaphigaster nebulosa (Poda, 1761) & $\boldsymbol{\Delta}$ \\
\hline \multicolumn{3}{|c|}{ Cydnidae } \\
\hline 157 & Canthophorus melanopterus melanopterus (Herrich-Schäffer 1835) & 口 \\
\hline
\end{tabular}

